\title{
Breaking Down the Problem: Optical Transitions, Electronic Structure, and Photoconductivity in Conjugated Polymer PCDTBT and in Its Separate Building Blocks
}

\author{
Natalie Banerji, ${ }^{*}, \dagger, \perp$ Eric Gagnon, ${ }^{\ddagger}$ Pierre-Yves Morgantini, ${ }^{\S}$ Sebastian Valouch, ${ }^{\dagger, \|}$ Ali Reza Mohebbi, ${ }^{\dagger}$ \\ Jung-Hwa Seo, ${ }^{\dagger}, \#$ Mario Leclerc, ${ }^{\ddagger}$ and Alan J. Heeger ${ }^{\dagger}$ \\ ${ }^{\dagger}$ Center for Polymers and Organic Solids, University of California at Santa Barbara, California 93106-5090, United States \\ ${ }^{\ddagger}$ Department of Chemistry, Université Laval, G1K 7P4 Quebec City, Quebec, Canada \\ ${ }^{\S}$ Faculté des sciences, Université de Genève, 30 Quai Ernest-Ansermet, CH-1211 Geneva 4, Switzerland \\ "Light Technology Institute, Karlsruhe Institute of Technology, Kaiserstrasse 12, D-76131 Karlsruhe, Germany
}

\section{Supporting Information}

\begin{abstract}
Conjugated polymers with alternating electronwithdrawing and electron-donating groups along their backbone (donor-acceptor copolymers) have recently attracted attention due to high power conversion efficiency in bulk heterojunction solar cells. In an effort to understand how the bandgap in a typical donor-acceptor copolymer is reduced by internal charge transfer character and what the implications of this charge transfer are, we have synthesized the isolated repeat unit (CDTBT) of the photovoltaically highly successful
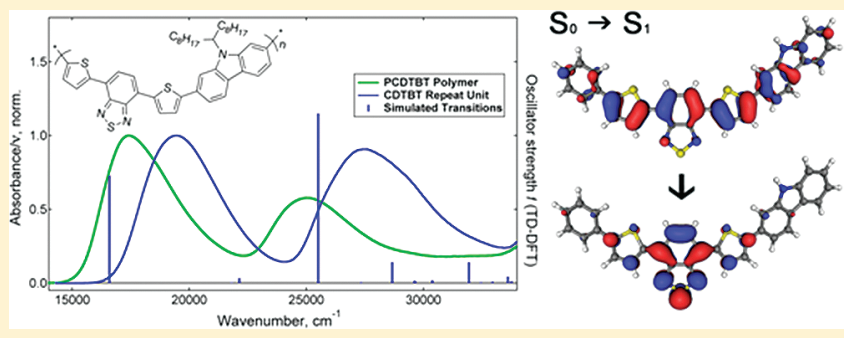
PCDTBT polymer. We compare here the spectroscopic and electrochemical properties of the polymer, the repeat unit, and the separate carbazole donor and dithienylbenzothiadiazole acceptor moieties (CB and ATBT, respectively) in the solid state and in solutions of various polarity. The results are interpreted with the help of time-dependent density functional theory (TD-DFT) calculations. We identify the dominant electronic transitions responsible for the first two absorption bands in the "camel back" spectrum of PCDTBT as partial charge transfer transitions with significant delocalization in the directly excited states. The low bandgap, overall shape, and partial charge transfer character of the PCDTBT absorption spectrum originate from transitions in the dTBT unit. The attached CB moiety extends the conjugation length in CDTBT, rather than acting as a localized donor. Further electronic delocalization, leading to a relatively small reduction in bandgap, occurs upon polymerization. We use our finding of higher delocalization following excitation in the second absorption band to explain the increased yield of photogenerated charges from this band in PCDTBT solid thin films. Moreover, we point out the importance of initial delocalization in the functioning of bulk heterojunction solar cells. The results presented here are therefore not only highly important for a better understanding of donor-acceptor copolymers in general but can also potentially guide the strategic development of future photovoltaic materials.
\end{abstract}

\section{INTRODUCTION}

Conjugated polymers have emerged as an important class of solution-processable materials, with a unique combination of mechanical, electrical, and optical properties for use in organic optoelectronics. ${ }^{1,2}$ Alternating donor-acceptor copolymers enjoy success as ambipolar charge transport materials ${ }^{3-5}$ and in highly efficient $(7-8 \%)$ bulk heterojunction $(\mathrm{BHJ})$ solar cells. $^{6-9}$ In contrast to a classic conjugated polymer such as P3HT (regioregular poly(3-hexylthiophene)), the repeat unit of a conjugated donor-acceptor polymer has a more complex molecular structure and consists of an electron-rich moiety (D) and an electron-poor moiety (A). Hybridization of the molecular orbitals from $\mathrm{D}$ and A leads to new transitions in the copolymer (which are believed to have intramolecular charge transfer character) and to a reduced bandgap. The low bandgap enables better harvesting of the solar spectrum in photovoltaic devices, especially in the near-infrared region. ${ }^{10,11}$ Moreover, it has been suggested that the $\mathrm{D}-\mathrm{A}$ alternation along the polymer backbone increases the double-bond character between the repeat units by stabilizing the quinoidal resonance form. ${ }^{10,12,13}$ The subsequent decrease in bond length alternation (suppression of Peierls instability) and increase in effective conjugation length (reduced twisting) also lower the bandgap.

Although electron delocalization along the backbone certainly plays an important role in defining the optical and electronic properties of conjugated polymers, the precise photophysics of even the classic polymers is generally not

Received: February 19, 2012

Revised: April 29, 2012

Published: May 7, 2012 
well understood. ${ }^{1,14,15}$ In particular, debate persists about the nature of the primary photoexcited species. There is increasing experimental evidence that this primary photoexcitation is highly delocalized along the polymer backbone, which might contribute to semiconductor-like behavior, and that it then relaxes to a more localized species on the ultrafast time scale. $^{16-22}$ Compared to the classic compounds, the photophysics of $\mathrm{D}-\mathrm{A}$ copolymers has been much less investigated. $^{23-25}$ The influence of the complex molecular structure in the large repeat unit and the precise origin of the typically broad and multiple absorption bands remain to be unambiguously elucidated. Moreover, the effect of the internal charge transfer character needs to be understood. Since charge transfer in optical transitions implies some localization of charges in the excited state, it is important to reconcile this notion with the concept of delocalization expected in conjugated systems.

Quantum-chemical simulations are often used to understand and predict the properties of D-A copolymers. ${ }^{26-36}$ To afford reasonable computational time, model oligomers of maximum 4 repeat units are investigated, and the results are extrapolated to account for the increased conjugation length of the polymers. Small molecule oligomers containing D and A units have also been synthesized on numerous occasions, often as photophysical model systems, ${ }^{37-40}$ to mimic photosynthesis, ${ }^{41-46}$ or for solution-processable BHJ solar cells. ${ }^{47-50}$ Nevertheless, there are only few examples where the $\mathrm{D}-\mathrm{A}$ oligomer corresponding to the repeat unit of an existing D-A copolymer has been prepared. ${ }^{13,51-59}$ To our knowledge, no detailed experimental study exists on the isolated repeat unit of an alternating D-A copolymer with high photovoltaic efficiency. Such an investigation would allow a crucial understanding of the intrinsic properties of the $\mathrm{D}-\mathrm{A}$ unit without the additional effects of extended conjugation. It could also provide direct experimental verification of theoretical results obtained with model oligomers.

Here, we have synthesized 4-(5-(N-(9-heptadecanyl)carbazol-2-yl)thiophen-2-yl)-7-(5-phenylthiophen-2-yl)benzo$[2,1,3]$ thiadiazole, abbreviated as CDTBT and depicted in Figure 1. This D-A dyad represents the repeat unit of the highly successful conjugated polymer PCDTBT (poly[N-9"heptadecanyl-2,7-carbazole-alt-5,5-(4',7'-di-2-thienyl-2',1',3'benzothiadiazole)), also shown in Figure 1. PCDTBT was first reported by Leclerc and co-workers. ${ }^{34,60,61}$ It turned out to perform best in photovoltaic devices within a series of 2,7carbazole copolymers, although other members of the family were predicted to have higher power conversion efficiency (PCE), based on their energy levels. ${ }^{62}$ Later, PCDTBT solar cells with over 6\% PCE and internal quantum efficiency approaching $100 \%$ were demonstrated. ${ }^{63}$ Recently, the PCE could be pushed further to $7.2 \%$, placing PCDTBT among the best polymeric solar cell materials available. ${ }^{64}$ We compare here the spectroscopic, electrochemical, and photoconductive properties of PCDTBT to the ones of isolated CDTBT. We also break down the problem to even smaller building blocks, the separate D and A moieties: CB (9-(heptadecan-9-yl)-9Hcarbazole) and dTBT (4,7-di(2-thienyl)-2,1,3-benzothiadiazole), respectively (see Figure 1). Experimental results in the solid state (thin films) and in solutions of various polarity are presented. Their interpretation is complemented by (timedependent) density functional theory ((TD)-DFT) calculation. Valuable insight was gained about the origin of the electronic transitions responsible for the absorption bands as well as about

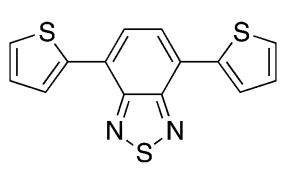

dTBT

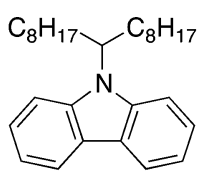

CB
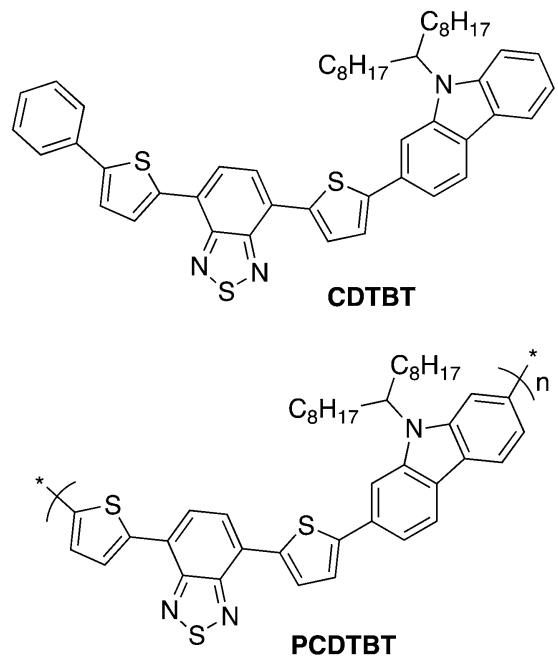

Figure 1. Molecular structures of the investigated compounds.

the delocalization of electron density and the charge transfer character in the ground and excited states. This insight is relevant for a better understanding of D-A copolymers in general and can potentially guide the strategic development of future materials.

\section{EXPERIMENTAL SECTION}

2.1. Materials. The PCDTBT polymer was synthesized by St-Jean Photochimie Inc. $\left(M_{\mathrm{n}}=39000, M_{\mathrm{w}}=104000, \mathrm{PDI}=\right.$ 2.7 ), according to the method previously described. ${ }^{34,60}$ Both $\mathrm{CB}^{65}$ and $\mathrm{dTBT}^{59}$ were also prepared using published procedures. The synthesis of CDTBT is summarized in Scheme 1 and described in detail below. The ${ }^{1} \mathrm{H}$ NMR spectra were recorded on a Varian AS400 apparatus in appropriate deuterated solvents at $298 \mathrm{~K}$. Chemicals shifts are reported as $\delta$ values (ppm), calibrated using the residual solvent peak.

4-(5-Bromothien-2-yl)-7-(thien-2-yl) benzo[2, 1,3]thiadiazole (2). 4,7-Di(2-thienyl)-2,1,3-benzothiadiazole ${ }^{59}$ (1, $1.50 \mathrm{~g}, 4.99 \mathrm{mmol}$ ) was dissolved in $215 \mathrm{~mL}$ of $\mathrm{CH}_{2} \mathrm{Cl}_{2}$ and 215 $\mathrm{mL}$ of $\mathrm{AcOH}$ and kept in the dark. $\mathrm{N}$-Bromosuccinimide $(0.978$ g, $5.49 \mathrm{mmol}$ ) was added in small portions over $30 \mathrm{~min}$. The reaction mixture was stirred at room temperature overnight. The mixture was then poured into $450 \mathrm{~mL}$ of water, and 300 $\mathrm{mL}$ of $\mathrm{CH}_{2} \mathrm{Cl}_{2}$ was added. The organic phase was separated and washed with water, brine, dried with anhydrous $\mathrm{MgSO}_{4}$, filtered, and evaporated to dryness. Recrystallization from $\mathrm{PhMe} / \mathrm{EtOH}$ afforded $0.71 \mathrm{~g}$ of a mixture enriched with the undesired dibrominated product; the mother liquor was evaporated to dryness, yielding $1.25 \mathrm{~g}$ of a $4: 1$ mixture of the desired product and the starting material, as determined by NMR spectroscopy. This mixture was used without further purification for the next step.

4-(5-Phenylthien-2-yl)-7-(thien-2-yl)benzo[2,1,3]thiadiazole (3). The crude mixture containing 4-(5-bromothien-2-yl)-7-(thien-2-yl)benzo[2,1,3] thiadiazole (2, $0.96 \mathrm{~g}, 2.5$ $\mathrm{mmol}$ ) and 4,7-di(2-thienyl)-2,1,3-benzothiadiazole (1, $0.19 \mathrm{~g}$, 


\section{Scheme 1. Overall Synthetic Route for CDTBT}

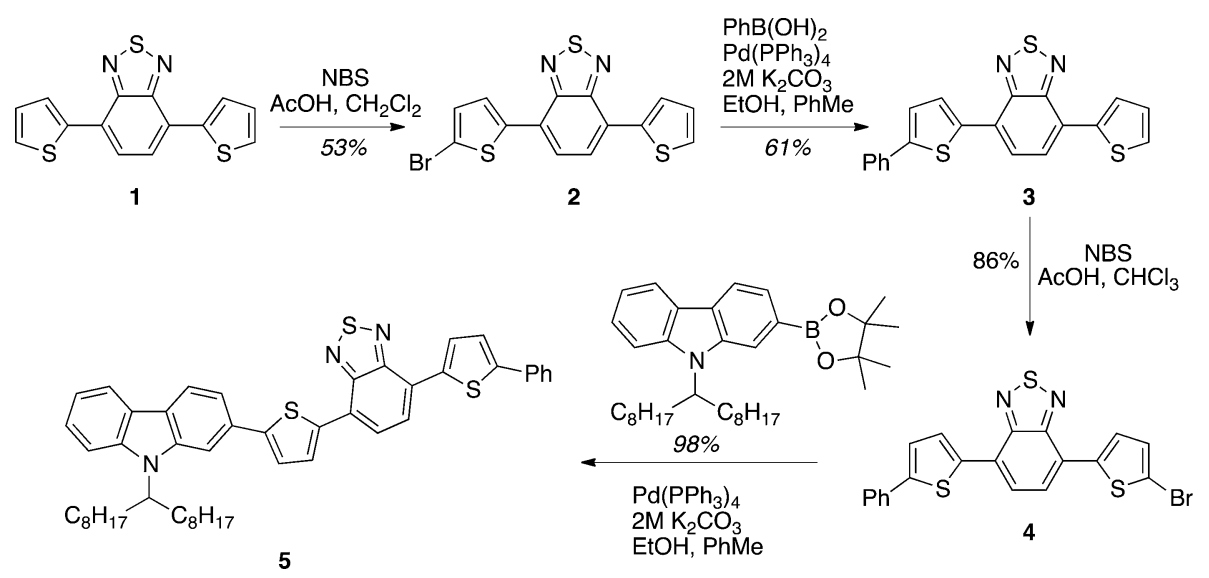

$0.63 \mathrm{mmol})$ was combined with an excess of phenylboronic acid (0.771 g, $6.33 \mathrm{mmol})$ and $\mathrm{Pd}\left(\mathrm{PPh}_{3}\right)_{4}(0.183 \mathrm{~g}, 0.158$ $\mathrm{mmol})$ in a mixture of $2 \mathrm{M} \mathrm{K}_{2} \mathrm{CO}_{3}(5 \mathrm{~mL}), \mathrm{EtOH}(5 \mathrm{~mL})$, and toluene $(10 \mathrm{~mL})$. The mixture was purged thoroughly with argon and then heated at $90{ }^{\circ} \mathrm{C}$ for $1 \mathrm{~h} 15$. After cooling to room temperature, the mixture was diluted with toluene and washed with water, brine, dried with anhydrous $\mathrm{MgSO}_{4}$, and filtered. The resulting solution was passed through a plug of silica gel, eluted with $\mathrm{PhMe}$, and evaporated to dryness. The crude product was recrystallized from $\mathrm{PhMe} / \mathrm{EtOH}$ to afford the title product as red flakes $(0.581 \mathrm{~g}, 1.54 \mathrm{mmol}, 61 \%) ; \mathrm{mp} 168-170$ ${ }^{\circ} \mathrm{C}$ (from $\left.\mathrm{PhMe} / \mathrm{EtOH}\right) .{ }^{1} \mathrm{H}$ NMR $\left(400 \mathrm{MHz}\right.$, acetone- $\left.d_{6}\right) \delta$ $8.22(\mathrm{~m}, 2 \mathrm{H}), 8.11\left(1 \mathrm{H}, \mathrm{d},{ }^{3} \mathrm{~J}=7.6 \mathrm{~Hz}\right), 8.08\left(1 \mathrm{H}, \mathrm{d},{ }^{3} J=7.6\right.$ $\mathrm{Hz}), 7.79\left(2 \mathrm{H}, \mathrm{d},{ }^{3} J=7.7 \mathrm{~Hz}\right), 7.65(1 \mathrm{H}, \mathrm{d}, 5.1 \mathrm{~Hz}), 7.60(1 \mathrm{H}$, d, $\left.{ }^{3} \mathrm{~J}=3.9 \mathrm{~Hz}\right), 7.45\left(2 \mathrm{H}, \mathrm{dd},{ }^{3} \mathrm{~J}=7.7,7.4 \mathrm{~Hz}\right), 7.34\left(1 \mathrm{H}, \mathrm{t},{ }^{3} \mathrm{~J}=\right.$ $7.4 \mathrm{~Hz}), 7.25(1 \mathrm{H}, \mathrm{dd}, J=5.1,3.9 \mathrm{~Hz})$. HRMS: calculated for $\mathrm{C}_{20} \mathrm{H}_{12} \mathrm{~N}_{2} \mathrm{~S}_{3}$ : 376.0163; found: 376.0156 .

4-(5-Phenylthien-2-yl)-7-(5-bromothien-2-yl)benzo[2, 1,3]thiadiazole (4). 4-(5-Phenylthien-2-yl)-7-(thien-2-yl)benzo$[2,1,3]$ thiadiazole $(3,0.500 \mathrm{~g}, 1.33 \mathrm{mmol})$ was dissolved in a mixture of $10 \mathrm{~mL}$ of $\mathrm{CHCl}_{3}$ and $10 \mathrm{~mL}$ of $\mathrm{AcOH}$, cooled at 0 ${ }^{\circ} \mathrm{C}$, and kept in the dark. N-Bromosuccinimide (0.249 g, 1.40 mmol) was added in one portion, and the mixture was stirred from $0{ }^{\circ} \mathrm{C}$ to room temperature over $6 \mathrm{~h}$. The solution was diluted with $\mathrm{CHCl}_{3}$, washed with water, brine, dried with anhydrous $\mathrm{MgSO}_{4}$, filtered, and evaporated to dryness. The crude product was recrystallized from $\mathrm{PhMe} / \mathrm{EtOH}$ to afford the title product as a red powder $(0.520 \mathrm{~g}, 1.14 \mathrm{mmol}, 86 \%)$; mp $180-182{ }^{\circ} \mathrm{C}$ (from $\left.\mathrm{PhMe} / \mathrm{EtOH}\right) .{ }^{1} \mathrm{H}$ NMR $(400 \mathrm{MHz}$, acetone- $\left.d_{6}\right) \delta 8.23\left(1 \mathrm{H}, \mathrm{d},{ }^{3} \mathrm{~J}=3.9 \mathrm{~Hz}\right), 8.11(2 \mathrm{H}, \mathrm{s}), 7.96(1 \mathrm{H}$, d, $\left.{ }^{3} \mathrm{~J}=3.9 \mathrm{~Hz}\right), 7.78\left(2 \mathrm{H}, \mathrm{d},{ }^{3} \mathrm{~J}=7.7 \mathrm{~Hz}\right), 7.60\left(1 \mathrm{H}, \mathrm{d},{ }^{3} J=3.9\right.$ $\mathrm{Hz}), 7.46\left(2 \mathrm{H}, \mathrm{dd},{ }^{3} J=7.7,7.4 \mathrm{~Hz}\right), 7.35\left(\mathrm{t}, 1 \mathrm{H},{ }^{3} \mathrm{~J}=7.4 \mathrm{~Hz}\right)$, $7.30\left(1 \mathrm{H}, \mathrm{d},{ }^{3} \mathrm{~J}=3.9 \mathrm{~Hz}\right)$. HRMS: calculated for $\mathrm{C}_{20} \mathrm{H}_{11} \mathrm{Br}_{1} \mathrm{~N}_{2} \mathrm{~S}_{3}$ : 453.9268; found: 453.9261 .

4-(5-(N-(9-heptadecanyl)carbazol-2-yl)thiophen-2-yl)-7(5-phenylthiophen-2-yl)benzo[2,1,3]thiadiazole (5). 4-(5Phenylthiophen-2-yl)-7-(5-bromothien-2-yl)benzo[2,1,3]thiadiazole (4, $0.257 \mathrm{~g}, 0.564 \mathrm{mmol}), 2-\left(4^{\prime}, 4^{\prime}, 5^{\prime}, 5^{\prime}\right.$-tetramethyl$1^{\prime}, 3^{\prime}, 2^{\prime}$-dioxaborolan-2'-yl)-N-9'-heptadecanylcarbazole ${ }^{65}(0.300$ g, $0.564 \mathrm{mmol})$, and $\mathrm{Pd}\left(\mathrm{PPh}_{3}\right)_{4}(0.033 \mathrm{~g}, 0.0282 \mathrm{mmol})$ were combined with $2 \mathrm{M} \mathrm{K}_{2} \mathrm{CO}_{3(\mathrm{aq})}(5 \mathrm{~mL}), \mathrm{EtOH}(2.5 \mathrm{~mL})$, and toluene $(10 \mathrm{~mL})$. The mixture was purged with argon and then heated at $90{ }^{\circ} \mathrm{C}$ for $4 \mathrm{~h}$. The solution was then diluted with toluene, washed with water and brine, dried with anhydrous $\mathrm{MgSO}_{4}$, filtered, and evaporated to dryness. The crude product was purified using flash chromatography (silica gel, 25\%
$\mathrm{CH}_{2} \mathrm{Cl}_{2}$ in hexanes) to afford the title product as a purple crystalline solid $(0.430 \mathrm{~g}, 0.551 \mathrm{mmol}, 98 \%)$; mp $94-96{ }^{\circ} \mathrm{C} .{ }^{1} \mathrm{H}$ NMR (600 MHz, $\mathrm{CDCl}_{3}$ ): 8.18 (br, 1H), 8.11 (br, 3H), 7.90 $(\mathrm{m}, 3 \mathrm{H}), 7.72(\mathrm{~m}, 3 \mathrm{H}), 7.58(\mathrm{br}, 1 \mathrm{H}), 7.50(\mathrm{br}, 1 \mathrm{H}), 7.43(\mathrm{~m}$, $4 \mathrm{H}), 7.32(\mathrm{t}, J=9.0 \mathrm{~Hz}, 1 \mathrm{H}), 7.22(\mathrm{~m}, 1 \mathrm{H}), 4,65(\mathrm{~m}, 1 \mathrm{H}), 2.34$ $(\mathrm{m}, 2 \mathrm{H}), 1.97(\mathrm{~m}, 2 \mathrm{H}), 1.5(\mathrm{~m}, 24 \mathrm{H}), 0.80(\mathrm{t}, J=6.0 \mathrm{~Hz}, 6 \mathrm{H})$. HRMS: calculated for $\mathrm{C}_{49} \mathrm{H}_{53} \mathrm{~N}_{3} \mathrm{~S}_{3}$ : 780.3474; found: 780.3483 .

2.2. Steady-State Spectroscopy. Dilute solutions (maximum visible absorbance $0.1-0.2$ ) were prepared of $\mathrm{CB}, \mathrm{dTBT}$, CDTBT, and PCDTBT in a $1 \mathrm{~cm}$ quartz cell for steady-state measurements. The solvents were pentane (PEN), octane (OCT), decane (DEC), toluene (TOL), diisopropyl ether (dipET), tert-butyl methyl ether (tbmET), octyl ether (oET), chloroform (CF), chlorooctane (CO), chlorobenzene (CB), ethyl acetate (ETAC), tetrahydrofuran (THF), dichloromethane $(\mathrm{DCM})$, dichlorobutane $(\mathrm{DCBu}), o$-dichlorobenzene (DCB), dichloroethane (DCE), butanol $(\mathrm{BuOH})$, isopropanol (iPrOH), acetone (ACE), ethanol (EtOH), methanol $(\mathrm{MeOH})$, acetonitrile (ACN), and dimethyl sulfoxide (DMSO). They were of highest commercially available purity (usually anhydrous) and were used as received without further purification. Thin films of CDTBT were spin-coated from chloroform $(7 \mathrm{mg} / \mathrm{mL})$ at $1000 \mathrm{rpm}$ in a nitrogen glovebox. PCDTBT was dissolved in a $1: 3(\mathrm{v}: \mathrm{v})$ solvent mixture of CB/ $\mathrm{DCB}$ at $7 \mathrm{mg} / \mathrm{mL}$ and spin-coated at $3000 \mathrm{rpm}$. Quartz or sapphire substrates were used.

Absorption spectra were recorded using a Beckman Coulter DU800 UV/vis spectrophotometer. Fluorescence emission spectra in solution were obtained with a Photon Technology International fluorimeter in a $90^{\circ}$ configuration under ambient conditions (there were no signs of degradation). The excitation wavelength was $300 \mathrm{~nm}$ for $\mathrm{CB}, 450 \mathrm{~nm}$ for dTBT and CDTBT, and $500 \mathrm{~nm}$ for PCDTBT. Thin film fluorescence spectra were recorded with a fiber-coupled CCD spectrometer (Ocean Optics HR4000) inside an integrating sphere (Labsphere, Spectraflect coating, $100 \mathrm{~mm}$ diameter, 4P-GPS-040$\mathrm{SF})$. The sample was excited with a $405 \mathrm{~nm}$ diode laser, and the direct emission and stray excitation light from the point of excitation were shielded from the fiber port with a baffle. To avoid degradation during the measurement, the integrating sphere was purged with nitrogen. Self-absorption of the thin films was subtracted using a method proposed by de Mello et al. $^{66}$

All emission spectra in film and solution were corrected for the wavelength-dependent sensitivity of the detection. For the emission spectra on the wavenumber scale $\left(\right.$ in $\mathrm{cm}^{-1}$ ), the 
fluorescence intensity was also multiplied by the square of the wavelength $\left(\lambda^{2}\right)$ to account for the bandpass constant in wavelength imposed by the monochromator of the fluorimeter. For a very rigorous comparison of the absorption and emission spectral band shapes, we divided the absorbance by the wavenumber and the emission by the cube of the wavenumber. This correction by the photon energy dependence is derived from the Einstein coefficients of absorption and spontaneous emission, and it is described in detail elsewhere. ${ }^{67}$ In this representation, the spectral intensity is directly proportional to the squared magnitude of the transition dipole moment (TDM) and as a consequence to the Franck-Condon factor. Note that the emission spectra in solution are cut below 12500 $\mathrm{cm}^{-1}(800 \mathrm{~nm})$ because of experimental limitations of the fluorimeter. The spectra on the wavelength scale without any corrections are shown in the Supporting Information, Figure S1.

2.3. Electrochemistry. Cyclic voltammetry (CV) experiments in dry o-dichlorobenzene (with $0.1 \mathrm{M}$ tetrabutylammonium tetrafluoroborate as supporting electrolyte) were performed for $\mathrm{CB}$, dTBT, CDTBT, and PCBTBT using a potentiostat/galvanostat Model 263A at a scan rate of $100 \mathrm{mV} /$ s. An $\mathrm{Ag} / \mathrm{Ag}^{+}$electrode, Pt wire, and a glassy carbon disk were used as the reference electrode, counter electrode, and working electrode, respectively. The data are reported versus the ferrocene/ferrocenium couple $\left(\mathrm{Fc} / \mathrm{Fc}^{+}\right)$. The $\mathrm{HOMO}$ and LUMO energies were deduced from the first onset reduction and oxidation potentials, respectively, using the HOMO level of ferrocene as a reference $\left(E_{\mathrm{HOMO}}=-4.8 \mathrm{eV}\right){ }^{68}$ unless states otherwise. In some cases, meausrements were also carried out in dry dichloromethane with tetrabutylammonium hexafluorophosphate (see text).

2.4. Computational Methods. The geometries of $\mathrm{CB}$, dTBT, and CDTBT were fully optimized at the DFT level using the nonlocal hybrid Becke three-parameter Lee-YangParr (B3LYP) hybrid functional ${ }^{69}$ and the $6-31 \mathrm{G}^{* *}$ basis set as implemented in the Gaussian 03 package. ${ }^{70}$ We chose this computational method, since it has been previously successful in a purely computational study of PCDTBT-related oligomers with $1-4$ repeat units. ${ }^{27}$ Geometry optimizations of the $\mathrm{CB}$ and the dTBT molecule were restricted to the $C_{2 v}$ symmetry point group. The alkyl side chains on the $\mathrm{CB}$ were replaced by hydrogen atoms for the calculations in $\mathrm{CB}$ and CDTBT. TDDFT calculations of singlet-singlet and singlet-triplet excitation energies were performed at the same level of theory (B3LYP/6-31G**). Molecular orbitals were generated using Molekel 5.4. ${ }^{71}$ The dipole moment in the first excited state (following a vertical transition, prior to nuclear relaxation) was computed using the Gaussian 09 package. $^{72}$

2.5. Steady-State Photoconductivity Measurements. Photoconductivity measurements were carried out on films of CDTBT and PCDTBT spin-coated from chloroform and a 1:3 (v:v) solvent mixture of $\mathrm{CB} / \mathrm{DCB}$ at $7 \mathrm{mg} / \mathrm{mL}$ onto quartz substrates. A planar Auston switch configuration was used: 70 $\mathrm{nm}$ thick gold electrodes were thermally evaporated on the sample surface through a $50 \mu \mathrm{m}$ steel shadow mask inside a glovebox equipped with a metal evaporator, forming an active area of $50 \mu \mathrm{m} \times 1000 \mu \mathrm{m}$. After metal deposition, and in order to minimize oxidation, the samples were immediately loaded into a vacuum chamber with pressure lower than $10^{-6}$ mbar. The samples were then illuminated with a monochromatic light source consisting of a tungsten lamp attached to a monochromator. Light was modulated at $174 \mathrm{~Hz}$ using an optical chopper, and the wavelength-dependent output power of the setup was determined using a calibrated silicon photodetector. An electric field was applied to the sample via a picoammeter (Keithley, 487) and the resulting current measured with a lock-in amplifier (Stanford Research SR830).

\section{RESULTS AND DISCUSSION}

The steady-state spectra for CB, dTBT, CDTBT, and PCDTBT are compared for solvents of varying polarity and thin films in Figure 2. The dielectric constants for ACN, DCB,

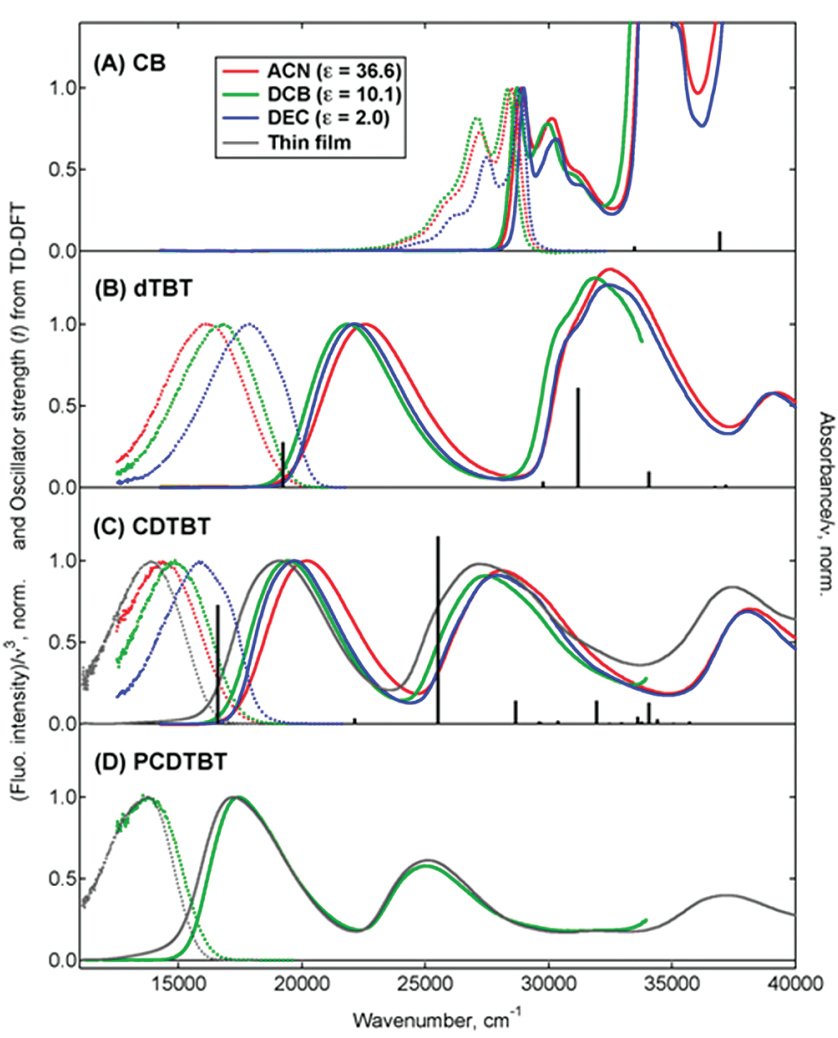

Figure 2. Steady-state spectra in TDM representation (see text) of $\mathrm{CB}$ (A), dTBT (B), CDTBT (C), and PCDTBT (D) recorded in solvents of various polarity (the dielectric constants are shown in the legend) and thin films. The dotted lines represent the emission spectra and the smooth lines the absorption spectra. The black vertical lines correspond to the TD-DFT simulated transitions (TD-B3LYP/6$\left.31 \mathrm{G}^{* * / / B} 3 \mathrm{LYP} / 6-31 \mathrm{G}^{* *}\right)$; their height represents the oscillator strength $(f)$. Note that transitions were calculated only below 35 $700 \mathrm{~cm}^{-1}$ in CDTBT.

and DEC are indicated in the legend. Note that sections 3.1-3.4 treat only the properties in highly dilute solution, where molecules are essentially isolated (negligible aggregation effects). In order to assign the absorption bands of CB, dTBT, and CDTBT, we performed TD-DFT calculations (for isolated molecules in the gas phase). This yields vertical transition energies $\left(E_{\mathrm{vert}}\right)$ between electronic states and their oscillator strength $(f)$, as listed together with the predominant contributions of one-electron transitions in Table S1 of the Supporting Information. The transitions are also plotted as black vertical lines in Figure 2 (the height is scaled to represent the oscillator strength), in comparison to experimental data. Finally, section 3.5 describes CDTBT and PCDTBT in the solid state. 
3.1. The CB Donor Unit. The CB molecule has the first absorption band near the ultraviolet range, as depicted in Figure 2A. It displays a pronounced vibronic structure with a sideband separation of about $1260 \mathrm{~cm}^{-1}$. The absorption maximum is in the $0-0$ band at 28740,28900 , and $28990 \mathrm{~cm}^{-1}$ in DCB, $\mathrm{ACN}$, and DEC, respectively. The structured fluorescence spectrum is close to the mirror image of the first absorption band. It is only very slightly Stokes shifted, the separation of the absorption and emission maxima being about $400 \mathrm{~cm}^{-1}$ in ACN and DCB and $170 \mathrm{~cm}^{-1}$ in DEC. The solvent-induced spectral shift in absorption and fluorescence is very weak and not correlated with solvent polarity. However, the shape of the spectra, in particular the width of the vibronic bands and their relative intensity, is slightly affected by the solvent.

From TD-DFT calculations, the $S_{0} \rightarrow S_{1}$ transition responsible for the first absorption band can be predominantly $(85 \%)$ assigned to a HOMO $\rightarrow$ LUMO transition (Table S1). In agreement with experiment, the oscillator strength is very weak (0.03), while transitions further in the ultraviolet range become more intense. The second absorption band can be attributed to the $S_{0} \rightarrow S_{2}$ transition, which has strong contributions from the HOMO-1 $\rightarrow$ LUMO and HOMO $\rightarrow$ LUMO +1 configurations ( $65 \%$ and $24 \%$, respectively). Since we found the calculated transition energies of CB to be strongly blue-shifted compared to the experiment (see Figure 2), we repeated the simulations with the TD-SAOP/TZ2P method following geometry optimization at the PW91PW91/DZVP level of theory. We obtained an excellent match between the theoretical and experimental absorption spectra (Supporting Information, Figure S2) and could confirm the above assignment of the $\mathrm{CB}$ absorption bands.

The shape of the above-mentioned orbitals is shown in Figure 3 together with the optimized geometry of CB. The frontier molecular orbitals as well as the HOMO-1 and LUMO +1 are dominated by $\pi$-orbitals that are delocalized over the entire molecule, so that very little charge transfer (redistribution of electron density) is to be expected during the $S_{0} \rightarrow S_{1}$ and $S_{0} \rightarrow S_{2}$ transitions. For the first transition, this is confirmed by the (TD)-DFT calculations, which predict a permanent dipole moment of $1.64 \mathrm{D}$ in the ground state and of $2.51 \mathrm{D}$ in the vertically excited state. The weak ground state dipole and weak dipole change during excitation explain the small solvatochromic shift of the absorption bands. Moreover, the small Stokes shift and mirror-image shape of the emission spectrum compared to the first absorption band point to a quite similar nature of the ground and emitting states; the emission is essentially the inverse of $S_{0} \rightarrow S_{1}$ absorption. A small displacement of the potential energy surfaces causes the observed vibrational progression. Overall, CB shows the typical photophysical behavior of small, rigid aromatic molecules in solution.

The calculated energy levels of the $\mathrm{CB}$ orbitals are represented as black lines in Figure 4. Note that to save computational time, the long alkyl chains attached to the nitrogen atom of CB have been replaced by a hydrogen atom, which should lead to only minor differences compared to the experimentally studied molecule. The HOMO level $(-5.33 \mathrm{eV})$ and LUMO level $(-0.64 \mathrm{eV})$ previously reported from a similar DFT simulation for a CB compound in which a methyl group was attached to the nitrogen are very comparable to our results. ${ }^{27}$ We also obtained the HOMO and LUMO levels experimentally (gray lines in Figure 4), from the cyclic voltammogram in DCB solution depicted in Figure S3 of the

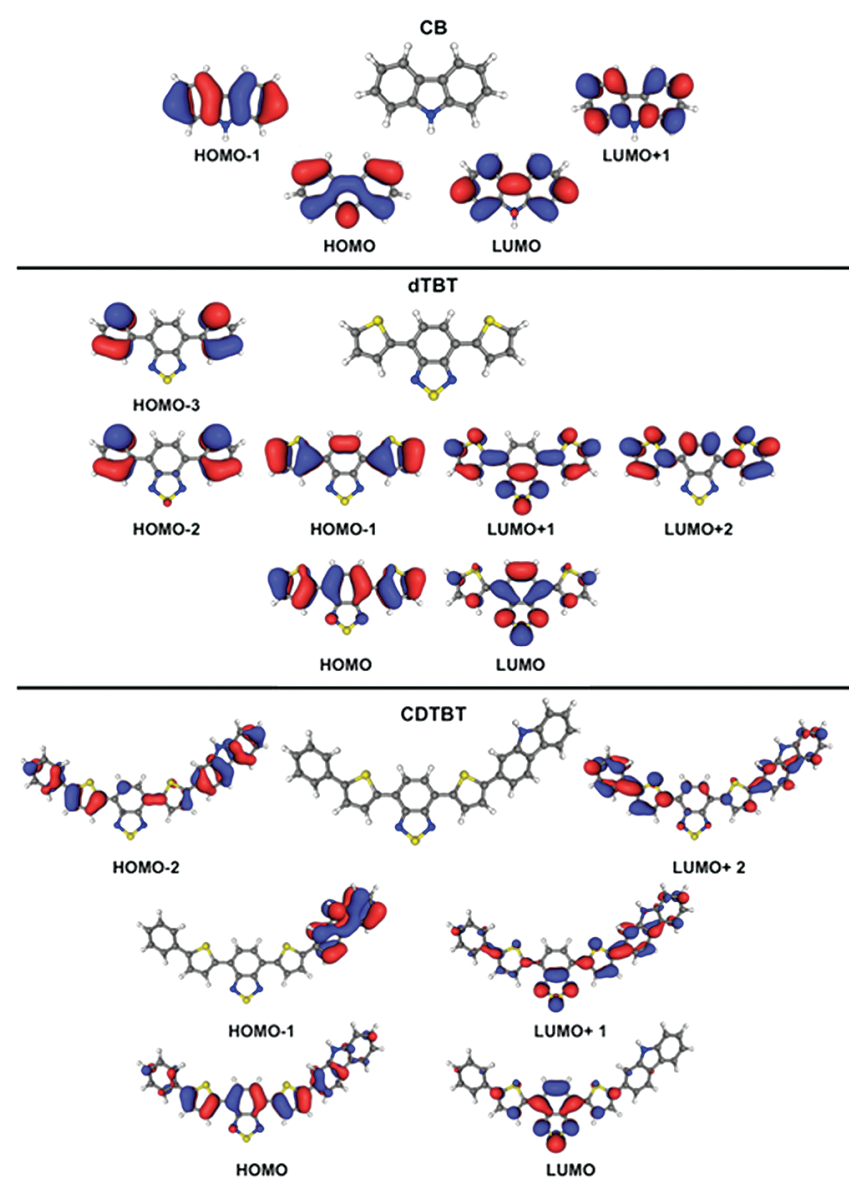

Figure 3. Optimized ground-state geometries and shape of selected molecular orbitals from DFT calculations (B3LYP/6-31G**) for CB, dTBT, and CDTBT.

Supporting Information and from the absorption edge. Two oxidation processes are observed in $\mathrm{CB}$, although the anodic peak position of the first one is not obvious. The corresponding half-wave potentials, the deduced frontier molecular orbital levels, and the first optical transition energy ("optical bandgap", $E_{\mathrm{g}}^{\mathrm{opt}}$, from the red edge of the absorption spectra in $\mathrm{DCB}$ ) are summarized in Table 1. The HOMO level of approximately $-5.22 \mathrm{eV}$ for $\mathrm{CB}$ is higher than the one of $-5.88 \mathrm{eV}$ found in the literature for a structurally very similar compound, ${ }^{73}$ but it was in both cases difficult to observe a clean oxidation peak. There is overall reasonable agreement between the energy levels experimentally estimated from the electrochemical potentials in the presence of a solvent environment and the gas-phase calculated data. As described in more detail elsewhere, ${ }^{27}$ simulations are limited by approximations in the computational methods. Calculated HOMO and LUMO levels are equivalent to the (negative) ionization potential $\left(I_{\mathrm{p}}\right)$ and electron affinity $\left(E_{\mathrm{a}}\right)$, respectively, only within certain approximations, where electron reorganization and vibronic coupling are neglected.

3.2. The dTBT "Acceptor" Unit. The dTBT unit is often considered to be the acceptor moiety of D-A copolymers such as PCDTBT. Strictly speaking, only the 1,2,3-benzothiadiazole (BT) acts as an electron withdrawer, and it is surrounded by two electron-donating thiophenes, so that dTBT has on overall $\mathrm{D}-\mathrm{A}-\mathrm{D}$ type structure. Isolated $\mathrm{BT}$ has been reported to absorb in the ultraviolet range around $300 \mathrm{~nm} \mathrm{(33330}$ $\left.\mathrm{cm}^{-1}\right),{ }^{74,75}$ while a single thiophene unit has its first transition 


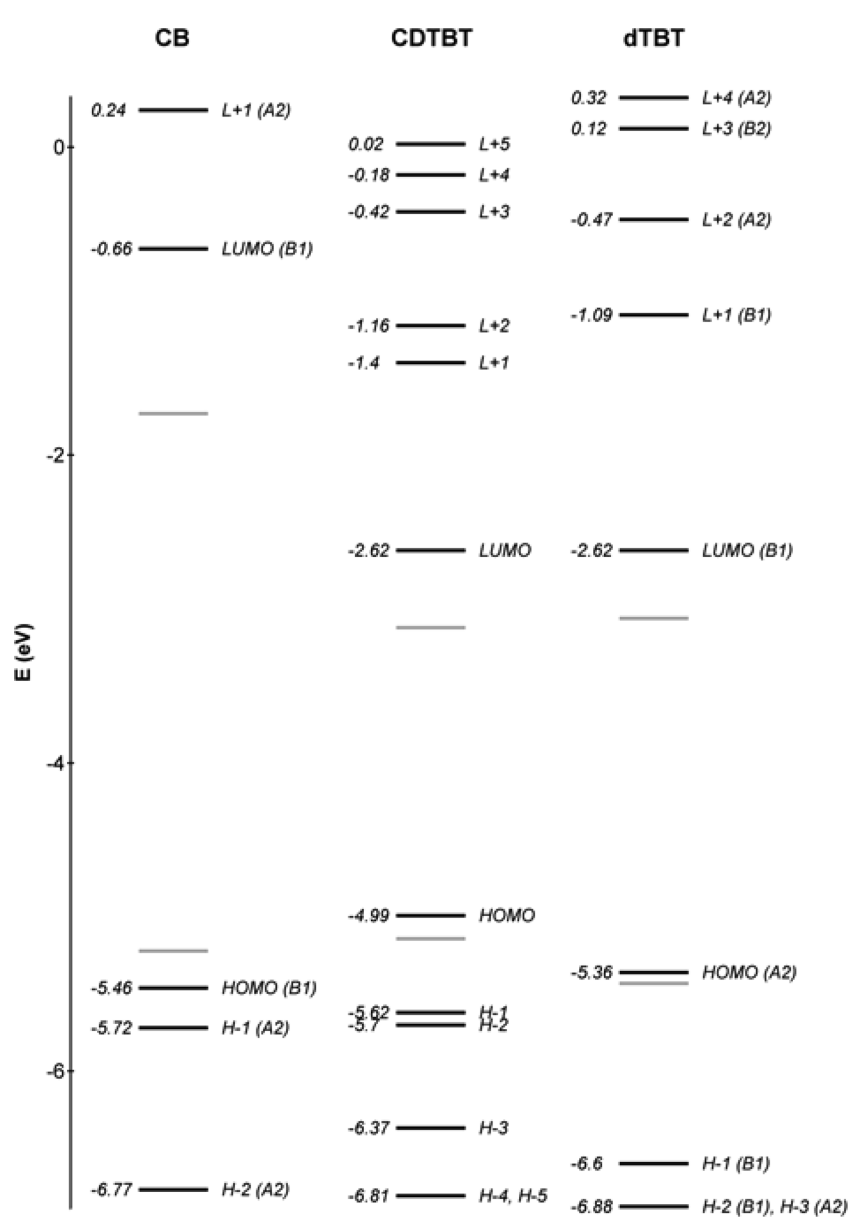

Figure 4. Energy levels of selected molecular orbitals (black lines) from DFT calculations (B3LYP/6-31G**) for CB, dTBT, and CDTBT. The designation ( $\mathrm{L}=\mathrm{LUMO}, \mathrm{H}=\mathrm{HOMO})$, energy and symmetry of the orbitals are also indicated (italic text). The HOMO and LUMO levels from cyclic voltammetry are shown for comparison as gray lines.

around $230 \mathrm{~nm}\left(43550 \mathrm{~cm}^{-1}\right)$ in the gas phase. ${ }^{76}$ The first absorption band of dTBT (around $22000 \mathrm{~cm}^{-1}$ with an absorption edge at $2.4 \mathrm{eV}$ ) is clearly red-shifted compared to both, as illustrated in Figure 2B. There are two more absorption bands in the shown window, around 32000 and $39000 \mathrm{~cm}^{-1}$. Except for a small shoulder in the second band, the three absorption bands are broad, structureless, and show no vibronic progression.

The calculated molecular orbitals of dTBT depicted in Figure 3 confirm that the individual electronic properties of BT and of the thiophenes are lost in $\mathrm{dTBT}$ due to strong electronic coupling (mixing) of the molecular orbitals. Both the HOMO and LUMO of dTBT have $\pi$-character and are delocalized over most of the almost planar molecule. The electron density of the HOMO is however on the long-axis backbone of the molecule (with very little density on the "exterior" thiadiazole ring, none on the sulfur atom), while the LUMO is concentrated on the entire BT unit (with less density on the thiophenes). The $\mathrm{HOMO}-1$, LUMO+1, and LUMO+2 all involve the thiophenes as well as either the benzene or the thiadiazole part of BT. Only the isoenergetic HOMO-2 and HOMO-3 bear the entire electron density on the thiophenes, with practically no contribution from BT. The calculated energy of the frontier orbitals (Figure 4, black lines) agrees again reasonably well with the experimental LUMO level obtained from the clearly defined quasi-reversible reduction wave (Figure S3) and with the HOMO level obtained by subtracting the optical bandgap. The electrochemical data are summarized in Table 1, and the energy levels are shown as gray lines in Figure 4.

Our TD-B3LYP calculations predict only the $S_{0} \rightarrow S_{1}$ transition in the vicinity of the first absorption band of dTBT (Figure 2B), so that we assign the band to this transition. The calculated $E_{\mathrm{vert}}$ coincides in fact with the onset rather than the maximum of the experimental spectrum, which we ascribe to the limitations of the DFT calculations (approximations leading for example to electron self-interaction effects, ${ }^{27}$ neglect of vibronic coupling, difficulty to reproduce charge transfer transitions, absence of solvent environment). The predominant contribution of the $S_{0} \rightarrow S_{1}$ transition in dTBT is from the HOMO $\rightarrow$ LUMO configuration (80\%, Table S1), which implies some redistribution of electron density from the conjugated backbone of the molecule toward the central BT unit (in particular, toward the thiadiazole ring located outside the backbone). The observed broad spectral features are typical for charge transfer transitions, as discussed in detail elsewhere. ${ }^{77}$ It is important to underline that this charge transfer character is only partial (both the HOMO and LUMO have some $\pi$ electron density delocalized over the entire molecule), as opposed to the case where an electron would be transferred entirely from the thiophenes to the BT.

The second absorption band of ATBT could in principle be due to a localized transition on the BT moiety, since it spectrally coincides with the first absorption band of isolated BT. ${ }^{74,75}$ Not surprisingly, TD-DFT calculations show that there are several transitions present within the broad envelope of the second absorption band (Figure $2 \mathrm{~B}$ and Table S1), which might explain why there is some structure (low-energy shoulder). The $S_{0} \rightarrow S_{5}$ transition is by far the most predominant one, with oscillator strength of 0.61 (about twice that of the $S_{0} \rightarrow S_{1}$ transition, in agreement with the experimental relative spectral intensities). This transition has a principal $(80 \%)$ contribution from the $\mathrm{HOMO} \rightarrow \mathrm{LUMO}+1$ configuration, which implies again partial electron redistrib-

Table 1. Onset $\left(E^{\text {onset }}\right)$ and Half-Wave $\left(E^{1 / 2}\right)$ Electrochemical Potentials, Frontier Orbital Energy Levels $\left(E_{\mathrm{HOMO}}, E_{\mathrm{LUMO}}\right)$ Estimated from $E^{\text {onset }}$ (Unless Stated Otherwise), Electrochemical Bandgap $\left(E_{\mathrm{g}}^{\text {elec }}=E_{\mathrm{LUMO}}-E_{\mathrm{HOMO}}\right.$ ), and First Optical Transition Energy ("Bandgap", $E_{\mathrm{g}}^{\mathrm{opt}}$ ) from the Absorption Edge for the Studied Compounds in DCB Solution

$\begin{array}{lclcccccc} & E_{\text {red1 }}^{\text {onset }}(\mathrm{V}) & E_{\text {red1 }}^{1 / 2}, E_{\text {red2 }}^{1 / 2}(\mathrm{~V}) & E_{\text {ox1 }}^{\text {onset }}(\mathrm{V}) & E_{\text {ox } 1,}^{1 / 2}, E_{\text {ox2 }}^{1 / 2}(\mathrm{~V}) & E_{\text {LUMO }}(\mathrm{eV}) & E_{\text {HOMо }}(\mathrm{eV}) & E_{\mathrm{g}}^{\text {elec }}(\mathrm{eV}) & E_{\mathrm{g}}^{\text {opt }}(\mathrm{eV}) \\ \text { CB } & & & & \sim 0.42,0.89 & -1.73^{a} & -5.22^{b} & 3.49 \\ \text { dTBT } & -1.74 & -1.85 & & & -3.06 & -5.43^{c} & \\ \text { CDTBT } & -1.68 & -1.77,-2.40 & 0.34 & 0.47, \sim 0.91^{d} & -3.12 & -5.14 & 2.02 \\ \text { PCDTBT } & \sim-1.41 & \sim-1.61,-1.98 & \sim 0.24 & \sim 0.43 & -3.39 & -5.04 & 1.65\end{array}$

${ }^{a}$ From $E_{\mathrm{HOMO}}+E_{\mathrm{g}}^{\mathrm{opt}} \cdot{ }^{b}$ From $E_{\mathrm{oxl}}^{1 / 2} \cdot{ }^{c}$ From $E_{\mathrm{LUMO}}-\mathrm{E}_{\mathrm{g}}^{\mathrm{opt}} \cdot{ }^{d}$ From a separate measurement in DCM. 
ution toward the thiadiazole ring, especially from the benzene of $\mathrm{BT}$, while significant electron density remains on the thiophenes during the transition (Figure 3). The 7\% HOMO$3 \rightarrow$ LUMO contribution to the $S_{0} \rightarrow S_{5}$ transition also involves charge transfer, this time from the thiophenes to the entire BT unit. Most of the other weak transitions simulated within the second absorption also implicate partial charge transfer, except the $S_{0} \rightarrow S_{6}$ one which involves orbitals that are more localized on the BT unit (LUMO, LUMO+4, HOMO4). It has however a relatively low oscillator strength (0.10). We conclude that the second absorption band of dTBT is dominated by partial charge transfer character and that it involves mixed orbitals with thiophene and BT contributions, rather than localized transitions on one of the subunits.

In spite of their partial charge transfer character, the absorption bands of dTBT hardly shift in different solvents. The first one has its maximum at $21860 \mathrm{~cm}^{-1}$ in DCB, 22120 $\mathrm{cm}^{-1}$ in DEC, and $22520 \mathrm{~cm}^{-1}$ in ACN (Figure 2B). The solvatochromic shift is the difference in the solvation energy between the initial and final state of the transition. This energy depends on several electrostatic interactions (dipole-dipole, dipole-induced dipole, Stark effect, dispersion) between the dissolved molecule and its solvent environment. We are particularly interested here in the dipole-dipole interactions, since information about the solute dipole moment (charge transfer character) in the ground and excited state can be extracted. We have used standard solvatochromic analysis, as reviewed extensively in the literature, ${ }^{78,79}$ and plotted the absorption maxima of $\mathrm{dTBT}$ (in TDM representation) against the Onsager polarity function $f\left(\varepsilon_{\mathrm{s}}\right)$ minus the function of the refractive index $f\left(n^{2}\right),\left[f\left(\varepsilon_{\mathrm{s}}\right)-f\left(n^{2}\right)\right]$, as defined:

$$
\begin{aligned}
& f\left(\varepsilon_{\mathrm{s}}\right)=2\left(\varepsilon_{\mathrm{s}}-1\right) / 2\left(\varepsilon_{\mathrm{s}}+1\right) \\
& f\left(n^{2}\right)=2\left(n^{2}-1\right) /\left(2 n^{2}+1\right)
\end{aligned}
$$

For solvents of comparable refractive index, this yields a linear correlation with a slope equal to $-\left(\vec{\mu}_{\mathrm{g}} \Delta \vec{\mu}_{\mathrm{abs}} / 4 \pi \varepsilon_{0} a^{3}\right)$, where $\vec{\mu}_{\mathrm{g}}$ is the permanent dipole moment in the ground state, $\Delta \vec{\mu}_{\text {abs }}$ is dipole moment difference vector between the ground and the directly excited state, $\varepsilon_{0}$ is the permittivity of free space, and $a$ is the radius of the solute molecule (assuming spherical shape). For the first two absorption bands of dTBT, we obtained a slope around $0 \mathrm{~cm}^{-1}$ (with some random scattering, as the used solvents had some variation in refractive index), indicating that $\left|\vec{\mu}_{\mathrm{g}} \Delta \vec{\mu}_{\mathrm{abs}}\right|$ is close to $0 \mathrm{D}^{2}$. This can be explained by the small dipole moment in the ground state $\left(\vec{\mu}_{\mathrm{g}}=0.13 \mathrm{D}\right.$ according to the DFT calculations). It is also consistent with a relatively small $\Delta \vec{\mu}_{\text {abs }}$ because the charge transfer is only partial. From the TD-DFT calculations, we estimate a dipole moment of $3.91 \mathrm{D}$ in the vertically excited $S_{1}$ state (before nuclear relaxation), leading to a moderate $\Delta \vec{\mu}_{\text {abs }}$ of $3.78 \mathrm{D}$. It should be noted here that the calculated $\vec{\mu}_{\text {g }}$ and $\Delta \vec{\mu}_{\text {abs }}$ are subject to the usual DFT limitations but are also very sensitive to conformational differences that exist between the DFT optimized gasphase geometry and the experimental geometry in solution. The simulations predict for example the thiophene sulfurs in anti conformation with respect to the thiadiazole ring. We cannot exclude rotation of the thiophenes in solution. Such a rotation has been shown to have minimal effect on the calculated energy levels but to increase the ground state dipole moment to $2.17 \mathrm{D}$ in the syn orientation. ${ }^{27}$ This is still relatively small and therefore not in contradiction with the weak dependence of the dTBT absorption spectrum on the solvent polarity. It has also been suggested that noncovalent interactions between the BT nitrogens and the thiophene sulfurs stabilize the molecule in the syn conformation and lead to its planarization. ${ }^{80}$

In order to estimate the interactions of dTBT with the solvent polarizability, the maxima of the first and second absorption bands of CDTBT were also plotted against $f\left(n^{2}\right)$. Unlike what was observed with solvent polarity, very good linear correlations are obtained with the solvent refractive index, with slopes of -4300 and $-4380 \mathrm{~cm}^{-1}$ and origins (estimates of the gas phase absorption maxima) of 23960 and $34040 \mathrm{~cm}^{-1}$. In this case, the interactions between the solute permanent dipole moment and the solvent induced dipole moment as well as the dispersion interactions (induced dipole of solute and solvent) are probed. Since the dependence on solvent dielectric constant showed no significant contribution of the dTBT permanent dipole to the absorption solvatochromism, we conclude that the latter is dominated by the dispersion interactions.

Finally, the strongly Stokes-shifted emission spectrum of dTBT is broad and structureless (Figure 2B). It therefore resembles the mirror image of the first absorption band. However, in contrast to absorption, the fluorescence spectrum shifts significantly to lower energy with increasing solvent polarity. The emission maximum goes from $17890 \mathrm{~cm}^{-1}$ in DEC, to $16750 \mathrm{~cm}^{-1}$ in DCB, and to $16100 \mathrm{~cm}^{-1}$ in ACN. This implies an increase of Stokes shift (difference between the absorption and emission maximum) from $4230 \mathrm{~cm}^{-1}$ in DEC, to $5090 \mathrm{~cm}^{-1}$ in DCB, and to $6420 \mathrm{~cm}^{-1}$ in ACN. We also note that the shape of the fluorescence spectrum is more asymmetric in DEC and a shoulder can be guessed on the high-energy side of the band. In the more polar solvents, the spectrum becomes more symmetrical and completely structureless. A clear linear correlation is found when the emission maximum is plotted against $\left[f\left(\varepsilon_{s}\right)-f\left(n^{2}\right)\right]$. The origin $\left(17880 \mathrm{~cm}^{-1}\right)$ represents the emission maximum without solvent (gas phase), while the slope in Joules is equal to $-\vec{\mu}_{\mathrm{e}} \Delta \vec{\mu}_{\mathrm{emi}} / 4 \pi \varepsilon_{0} a^{3}$. Here, $\vec{\mu}_{\mathrm{e}}$ is the permanent dipole moment vector of dTBT in the emitting state (not necessarily equal to the directly excited state if there is relaxation) and $\Delta \vec{\mu}_{\text {emi }}$ is the difference in dipole between the emitting and ground states. By estimating the radius $a$ from the van der Waals increment method, ${ }^{81}$ a value for $\left|\vec{\mu}_{\mathrm{e}} \Delta \vec{\mu}_{\text {emi }}\right|$ of 3.52 $\times 10^{-58} \mathrm{C}^{2} \mathrm{~m}^{2}\left(31.6 \mathrm{D}^{2}\right)$ is obtained. Using $\vec{\mu}_{\mathrm{g}}=0.13 \mathrm{D}$ from DFT and assuming parallel vectors, this equation can be solved to yield $\vec{\mu}_{\mathrm{e}}=5.7 \mathrm{D}$, which corresponds to a relatively high dipole in the emitting state. The large experimental Stokes shift between the first absorption and the emission band (Figure 2) points to considerable relaxation in the excited state (conformational and solvation). Therefore, it is probable that the charge transfer character in emission is stronger than the one in absorption, meaning that the directly excited state has a smaller dipole moment than the relaxed emitting state (as confirmed by the calculated $3.78 \mathrm{D}$ value in the vertical $S_{1}$ state).

3.3. The Linked CDTBT Repeat Unit. It is obvious that the absorption spectrum of CDTBT in solution is not a composite of the ones its separate units, $\mathrm{CB}$ and $\mathrm{dTBT}$ (Figure $2 \mathrm{C})$. It rather resembles a red-shifted version of the dTBT spectrum, with three broad bands around 20000,28000 , and $38000 \mathrm{~cm}^{-1}$. There are no obvious transitions due to the $\mathrm{CB}$ moiety in the shown window. Nevertheless, the presence of this group changes the relative intensity and energy of the absorption bands compared to dTBT. The shape of the first absorption band of CDTBT is very similar to the one of dTBT. 
It has an absorption edge at $2.1 \mathrm{eV}, 0.3 \mathrm{eV}$ lower than in dTBT. With a maximum of $19450 \mathrm{~cm}^{-1}$ in DCB, $19670 \mathrm{~cm}^{-1}$ in DCE, and $20180 \mathrm{~cm}^{-1}$ in ACN, its maximum is overall red-shifted by about $2400 \mathrm{~cm}^{-1}$ in each solvent. The band is narrower and more asymmetric (with a very weak shoulder on the low-energy side) in DEC compared to ACN. The second absorption band of CDTBT in solution shows more differences compared to the corresponding band in dTBT. It is very red-shifted (by about $4400 \mathrm{~cm}^{-1}$ at the maximum), and its relative intensity is much lower, making it slightly weaker than the first absorption band. Moreover, its shape is less structured, with a very weak shoulder on the high-energy side (more visible on the wavelength scale, Figure S1), while the more pronounced shoulder in ATBT is on the low-energy side. Finally, the maximum of the third absorption band is separated by only $1100 \mathrm{~cm}^{-1}$ in CDTBT and dTBT. The relative intensity of this band compared to the first absorption band is higher in CDTBT. The emission spectrum of CDTBT consists of a broad band, close to a redshifted mirror image of the first absorption band in all three solvents (Figure 2C). There is a high-energy shoulder in nonpolar DEC, which vanishes in the more symmetrical spectra recorded in the higher polarity solvents. The emission maximum is found at 15870,14860 , and $14350 \mathrm{~cm}^{-1}$ in DEC, DCB, and ACN, respectively. Thus, the Stokes shift is important and it increases with solvent polarity from $3790 \mathrm{~cm}^{-1}$ in DEC, to $4410 \mathrm{~cm}^{-1}$ in DCB, and to $5830 \mathrm{~cm}^{-1}$ in ACN. With respect to dTBT, the emission spectrum of CDTBT has a similar shape (though it is slightly narrower) and displays a smaller Stokes shift in all solvents. The emission band maximum in CDTBT compared to dTBT is red-shifted by $1750 \mathrm{~cm}^{-1}$ in ACN and by $2010 \mathrm{~cm}^{-1}$ in DEC, which is less than the shift in the first absorption band.

As discussed above, the overall shape of the CDTBT steadystate spectra suggests that the optical transitions are strongly dominated by dTBT (Figure 2). Judging from the shifted band positions, they are however not entirely localized on the dTBT unit. We used again DFT simulations and cyclic voltammetry to understand how the electronic structures of $\mathrm{CB}$ and $\mathrm{dTBT}$ interact when they are covalently linked to form CDTBT. Note that the CDTBT calculations also include an additional phenyl $\mathrm{Ph})$ group attached at the extremity of the dTBT moiety, since it was present in the experimental compound for synthetic reasons. Removing it had no significant effect on the DFT results.

Because of the relatively complex structure of CDTBT, the TD-DFT calculations predict many transitions in the calculated $<35700 \mathrm{~cm}^{-1}$ range, but the $S_{0} \rightarrow S_{1}$ transition near the onset of the first absorption band and the $S_{0} \rightarrow S_{4}$ transition near the onset of the second absorption band are clearly predominant, with oscillator strengths of 0.73 and 1.15 , respectively (Figure 2 and Table S1). The $S_{0} \rightarrow S_{1}$ transition in CDTBT is mainly due to a HOMO $\rightarrow$ LUMO contribution (83\%), making it very similar to the $S_{0} \rightarrow S_{1}$ transition in dTBT. Indeed, the LUMOs in the two compounds are very similar in shape and energy (Figures 3 and 4); i.e., the LUMO of CDTBT is localized strongly on the dTBT unit. The HOMO of CDTBT also has a similar shape as the one of dTBT, but the conjugation extends now over the entire backbone of the molecule, including the $\mathrm{Ph}$ and $\mathrm{CB}$ groups. Judging from the shape, symmetry, and energy of the orbitals, it results predominately from the coupling of the HOMO of dTBT and the HOMO-1 of CB (Figures 3 and 4). The ensuing energy level is higher (less negative) than the parent ones, leading to the observed reduction in energy of the
HOMO $\rightarrow$ LUMO transition (red-shift of the first absorption band). The calculated oscillator strength of the $S_{0} \rightarrow S_{1}$ transition in CDTBT is increased 2.6 times compared to dTBT, probably because the transition dipole moment (oriented along the long axis of the molecule) ${ }^{27}$ is enhanced by the extended conjugation in the HOMO. The cyclic voltammogram of CDTBT (Figure S3) shows two reduction processes and an oxidation. A second oxidation wave could be evidenced when the measurement was repeated in DCM solution. The redox potentials and deduced frontier molecular orbital levels are summarized in Table 1, and the latter are illustrated in Figure 4. Note that both the HOMO and LUMO level could be obtained entirely from electrochemistry (without using the optical bandgap) and that their difference (electrochemical bandgap, $\left.E_{\mathrm{g}}^{\mathrm{elec}}\right)$ matches well $E_{\mathrm{g}}^{\text {opt }}$. The experimental data confirm that the first reduction potential (LUMO level) is quite similar in $\mathrm{dTBT}$ and CDTBT, while the HOMO level is destabilized in CDTBT.

As expected, the $S_{0} \rightarrow S_{1}$ transition predicted by TD-DFT for CDTBT in the first absorption band (mainly HOMO $\rightarrow$ LUMO) has charge transfer character. Electron density concentrates on the BT moiety (especially on the thiadiazole ring) during absorption (Figure 3). Again, it must be stressed that this polarization in the excited state is only partial, and some $\pi$-delocalization is maintained even with the electron predominantly in the LUMO, also due to weak contributions of more delocalized electron configurations. A much stronger charge transfer occurs during the $S_{0} \rightarrow S_{2}$ transition, which is dominated by a transfer of the electron from the HOMO-1 (entirely on the CB) to the LUMO (almost entirely on the BT) (see Figure 3). However, the oscillator strength of the $S_{0} \rightarrow S_{2}$ transition is calculated to be only 0.003 due to the weak overlap of the involved orbitals (Table S1). The solvatochromism of the first absorption band of CDTBT resembles the one of dTBT. For a thorough analysis, we recorded the steady-state spectra of CDTBT in 23 solvents and plotted the first absorption maximum in TDM representation against $\left[f\left(\varepsilon_{\mathrm{s}}\right)-\right.$ $\left.f\left(n^{2}\right)\right]$ in Figure 5A. The ground-state dipole and its change during partial charge transfer absorption are not sufficient in CDTBT to cause a significant correlation of the first absorption maximum with the Onsager polarity function (slope close to zero, $\left.\left|\vec{\mu}_{\mathrm{g}} \Delta \vec{\mu}_{\mathrm{abs}}\right| \approx 0 \mathrm{D}^{2}\right)$. The calculated values for the dipoles $\left(\vec{\mu}_{\mathrm{g}}=1.98 \mathrm{D}\right.$ and $\left.\Delta \vec{\mu}_{\mathrm{abs}}=6.01 \mathrm{D}\right)$ appear too big in view of the experimental result, possibly due to solution conformations that are not accounted for. The gas-phase optimized geometry has a $26^{\circ}$ twist angle between the planes of the dTBT and CB moieties. We cannot exclude other conformations in solution (due to rotation around the single bonds adjacent to the thiophene groups, together with noncovalent stabilizations). In Figure 5B, the first absorption maximum of CDTBT is plotted against $f\left(n^{2}\right)$, and a very good linear correlation is obtained $\left(-3485 \mathrm{~cm}^{-1}\right.$ slope, $21190 \mathrm{~cm}^{-1}$ origin), showing that dispersion interactions with the solvent dominate the absorption transition.

The $S_{0} \rightarrow S_{4}$ transition prevailing in the second absorption band of CDTBT has 90\% HOMO $\rightarrow$ LUMO+1 character (from TD-DFT, Table S1). This involves some redistribution of electron density toward the thiadiazole ring, especially away from the BT central benzene (Figure 3), so that there is again partial charge transfer character. Nevertheless, some of the electron density also remains delocalized on the backbone in both predominantly involved orbitals, and this delocalization includes the $\mathrm{CB}$ unit. Except for this extension of the 

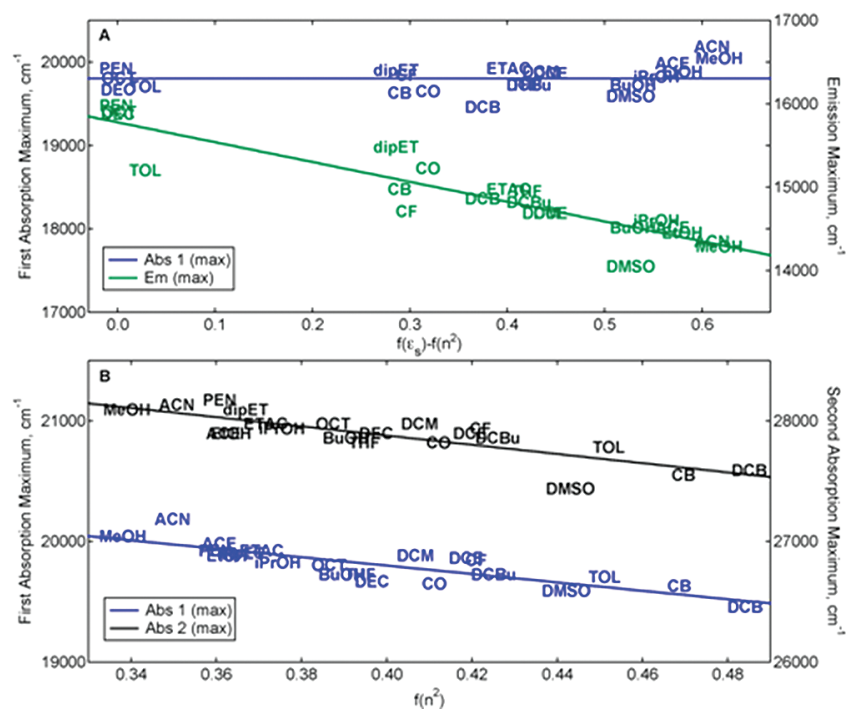

Figure 5. (A) First absorption and emission maxima of CDTBT in TDM representation versus the Onsager polarity function $\left[f\left(\varepsilon_{\mathrm{s}}\right)-\right.$ $\left.f\left(n^{2}\right)\right]$ (B) First and second absorption maxima of CDTBT in TDM representation versus the Onsager function of the solvent refractive index $f\left(n^{2}\right)$. The refractive index and dielectric constant for the used solvents are given in Table S2 of the Supporting Information.

conjugation toward $\mathrm{CB}$, the $\mathrm{S}_{0} \rightarrow \mathrm{S}_{4}$ transition in CDTBT resembles the $S_{0} \rightarrow S_{5}$ one in dTBT (mainly responsible for its second absorption band). The delocalization onto the $\mathrm{CB}$ unit increases the HOMO energy and decreases the LUMO+1 energy so that the transition energy is strongly reduced in CDTBT, more than for the $S_{0} \rightarrow S_{1}$ transition, where only the HOMO energy increases compared to dTBT. This explains the larger red-shift in the second than in the first absorption band when going from dTBT to CDTBT. Overall, the delocalization in the $S_{0} \rightarrow S_{4}$ transition of CDTBT is higher than the one in the $S_{0} \rightarrow S_{1}$ transition of this compound. The calculated oscillator strength of the $S_{0} \rightarrow S_{4}$ transition in CDTBT is increased 1.9 times compared to the $S_{0} \rightarrow S_{5}$ transition in dTBT. This increase in oscillator strength is less than the one predicted by TD-DFT for the $S_{0} \rightarrow S_{1}$ transition, accounting for the experimental apparent increase of the relative intensity of the first absorption band in CDTBT. As shown in Figure 5B, the second absorption maximum of CDTBT also correlates with $f\left(n^{2}\right)\left(-3810 \mathrm{~cm}^{-1}\right.$ slope, $29400 \mathrm{~cm}^{-1}$ origin, predominant dispersion interactions). The relative similarity of the shown slopes points to a comparable change of solute polarizability between the ground state and the directly excited state for both absorption bands. There are several additional transitions with weak oscillator strength calculated within the broad second absorption band of CDTBT, as shown in Figure 2. They might be responsible for the asymmetric band shape (high-energy shoulders). Most transitions involve orbitals that are delocalized over the entire molecule (for example, $\mathrm{HOMO}-2$, LUMO+1, and LUMO+2; see Figure 3 and Table S1) as well as some charge transfer (because the orbitals have slightly different electron distribution). We refrain here from assigning the third absorption band due to the large number of predicted transitions at high energy.

Finally, the emission spectrum in CDTBT is less Stokes shifted and less shifted than the first absorption band compared to dTBT. Nevertheless, the emission spectra of dTBT and CDTBT both strongly shift to the red with increasing solvent polarity (Figure 2). A reasonable linear dependence is obtained in Figure 5A, where the CDTBT emission maximum in 23 solvents is plotted as a function of $\left[f\left(\varepsilon_{s}\right)-f\left(n^{2}\right)\right]$, with an origin of $15780 \mathrm{~cm}^{-1}$ and a slope of $-2375 \mathrm{~cm}^{-1}$. Note that the scattering of the points in Figure 5A (especially in solvents such as TOL or DMSO with high refractive index) is mainly due to the fact that we isolate here the dipole-dipole interactions (solvent polarizability is not accounted for), although the used solvents do not have exactly the same refractive index. By estimating $a=5.58 \times 10^{-10} \mathrm{~m}$ from the van der Waals increment method, ${ }^{81}$ a value for $\vec{\mu}_{\mathrm{e}} \Delta \vec{\mu}_{\text {emi }} \mid$ of $9.12 \times 10^{-58} \mathrm{C}^{2}$ $\mathrm{m}^{2}\left(81.9 \mathrm{D}^{2}\right)$ is obtained from the slope for CDTBT. Using $\vec{\mu}_{\mathrm{g}}$ $=1.98 \mathrm{D}$ from the DFT calculations, we can estimate $\vec{\mu}_{\mathrm{e}}=10.1$ $D$. The dipole in the emitting state (the relaxed $S_{1}$ state) is therefore much higher in CDTBT than in dTBT. It is also higher than the $7.99 \mathrm{D}$ dipole obtained by TD-DFT for CDTBT in the vertical (nonrelaxed) $S_{1}$ state. To elucidate this further, we are currently investigating the excited-state relaxation in dTBT and CDTBT using ultrafast spectroscopy. Preliminary experimental evidence points indeed to an increase of the charge transfer character when going from the directly excited state to the emitting state in CDTBT.

3.4. From the Repeat Unit to the Polymer. From $M_{\mathrm{n}}=$ $39000 \mathrm{~g} / \mathrm{mol}$ for the used polymer batch, it can be estimated that there are on average 55 repeat units per PCDTBT chain. In general, polymerizing a conjugated repeat unit leads to splitting of the molecular orbitals as the conjugation length (electron delocalization in the involved $\pi$ orbitals) is increased. The HOMO level is destabilized and the LUMO level is stabilized, causing a reduction of bandgap (red-shift of the absorption spectrum). The density of states near the frontier orbitals also increases to finally yield energy bands rather than discrete energy levels. ${ }^{13}$ For example, a single thiophene unit absorbs around $230 \mathrm{~nm}^{76}$ while polythiophene (P3HT in solution) absorbs in the visible range with a bandgap around $520 \mathrm{~nm} .{ }^{16}$ The situation in PCDTBT is expected to be more complex, in particular due to the large size of the conjugated repeat unit (it contains five aromatic rings in contrast to one aromatic ring in most classic conjugated polymers).

Because of low solubility, the solution spectra of PCDTBT could only be recorded in DCB (Figure 2D). It can be seen that linking the CDTBT repeat units together during polymerization does not change the overall features of the absorption and emission spectra. There are again three predominant broad and structureless absorption bands (the third one is masked by the solvent) and a broad emission band. The trends observed when going from dTBT to CDTBT are continued upon polymerization: The first two absorption bands shift to the red (the second one more than the first), the relative intensity of the second absorption band decreases, and the Stokes shift becomes smaller. The first absorption band of PCDTBT is slightly narrower than the corresponding one in CDTBT. Its maximum at $17410 \mathrm{~cm}^{-1}$ is about $2000 \mathrm{~cm}^{-1}$ red-shifted in the polymer, while its absorption edge at $15390 \mathrm{~cm}^{-1}(1.9 \mathrm{eV})$ is $0.2 \mathrm{eV}$ lower than in CDTBT. Thus, the decrease in bandgap between CDTBT and PCDTBT is actually quite small (much less than when comparing $\mathrm{P} 3 \mathrm{HT}$ to its repeat unit). The maximum of the second absorption band of PCDTBT is about $2200 \mathrm{~cm}^{-1}$ at lower energy compared to CDTBT (Figure 2), its relative intensity is significantly reduced, it is much narrower, and it now has a completely symmetrical and structureless shape. The emission spectrum of PCDTBT peaks at 13810 $\mathrm{cm}^{-1}$, red-shifted compared to the first absorption maximum by 
$3590 \mathrm{~cm}^{-1}$ and compared to the CDTBT emission by $\sim 1000$ $\mathrm{cm}^{-1}$.

Quantum calculations can again be used to gain insight into what happens when CDTBT units are linked together. ${ }^{27,32} \mathrm{~A}$ previously reported computational study used the same TDDFT method (B3LYP/6-31G**) as the one employed here to simulate the optical transitions for oligomers with $n=1-4$ repeat units corresponding to $\mathrm{D}-\mathrm{A}$ copolymers with high photovoltaic efficiency, including PCDTBT. ${ }^{27}$ The simulations show that the HOMO level increases by about $0.15 \mathrm{eV}$ and the LUMO value decreases by about $0.08 \mathrm{eV}$ when incrementing the chain length from one to four units in the PCDTBT-based oligomer, due to extended conjugation. The overall shape of the frontier orbitals in the tetramer is still very similar to the one that we show for the CDTBT monomer in Figure 3. The HOMO is delocalized along the backbone of several repeat units (with no electron density on the exterior thiadiazole rings). The LUMO in the tetramer is more concentrated on the BT units (with significant density on the thiadiazoles). Its electron density is somehow delocalized over the chain, since it has probability of being on several of the BT units (not on a single one of them). However, the LUMO is only slightly stabilized upon oligomerization because of this partial localization on the BTs. Our CV data (Table 1 and Figure S3) confirm an increase in HOMO energy and decrease in LUMO energy in PCDTBT compared to CDTBT. The electrochemical orbital levels should be considered as estimates, since the observed two reduction and the oxidation waves were very weak due to low solubility of the polymer. Therefore, a lesser agreement between the optical and electrochemical bandgap is obtained (Table 1). Both the HOMO and LUMO energy of PCDTBT found here are also higher (less negative) than the ones of $E_{\mathrm{HOMO}}=-5.5 \mathrm{eV}$ and $E_{\mathrm{LUMO}}=-3.6 \mathrm{eV}$ reported in the literature. ${ }^{60}$ This could be due to the fact that the previous measurement was done on a different polymer batch, in the solid state instead of solution, and with a different apparatus.

Although a high density of states around the frontier molecular orbitals (above the LUMO and below the HOMO) is expected in the PCDTBT polymer, the authors of the previous TD-DFT calculations found only two dominant transitions in the visible range for the tetramer, which has already a very long conjugated backbone. ${ }^{27}$ It can therefore be deduced that the two low-energy absorption bands of PCDTBT are also dominated by those two transitions, even if there will be small contributions from other states within the energy bands. The $S_{0} \rightarrow S_{1}$ transition energy (first absorption band) simulated by TD-DFT for the PCDTBT oligomers decreases with chain length and considerably increases its oscillator strength. ${ }^{27}$ In agreement with our experimental observation in PCDTBT (Figure 2), the oscillator strength of the $S_{0} \rightarrow S_{32}$ transition (predominant in the second absorption band) is lower in the tetramer than the one of the $S_{0} \rightarrow S_{1}$ transition. The authors of the previous study found the $S_{0} \rightarrow S_{1}$ transition to have partial charge transfer character, with a predominant redistribution of electron density from the delocalized backbone toward the BT units. ${ }^{27}$ The transition resembles a more delocalized version of the corresponding transition in CDTBT, since the conjugated backbone is now more extended than in the repeat unit and several BT moieties are involved. The $S_{0} \rightarrow S_{32}$ transition in the tetramer (second absorption band) is also analogous to the corresponding $S_{0} \rightarrow$ $\mathrm{S}_{4}$ transition in CDTBT, with a predominant shift of electron density away from the benzene toward the thiadiazole part of the BT units (partial charge transfer), while considerable delocalization in all involved orbitals is also maintained. As the dominant features of the CDTBT transitions originate already from $\mathrm{dTBT}$ and the tetramer really is an extended version of $\mathrm{CDTBT}^{27}$ the strong resemblance between the absorption spectra of dTBT, CDTBT, and PCDTBT is explained.

All the separate segments of PCDTBT (CB, BT, and thiophene) absorb in the ultraviolet (200-300 nm), and it emerges from our results that the main reduction of bandgap occurs already in $\mathrm{dTBT}$, rather than when the CDTBT repeat unit is polymerized. Indeed, the bandgap of PCDTBT is about $1.9 \mathrm{eV}$ lower than the one of isolated BT. $75 \%$ of the bandgap is already reduced in $\mathrm{dTBT}, 15 \%$ more when $\mathrm{CB}$ is linked to dTBT to form the repeat unit, and only $10 \%$ more upon polymerization. The relatively small decrease in bandgap (0.2 $\mathrm{eV}$ in solution) from CDTBT to PCDTBT is consistent with the reported fast saturation of frontier orbital energy and $S_{0} \rightarrow$ $S_{1}$ transition energy calculated for increasing oligomer length. ${ }^{27}$ Oligomers corresponding to other investigated D-A copolymers showed a much stronger decrease of bandgap with increasing chain length, which the authors ascribed to the longer conjugation path length in the PCDTBT repeat unit. Indeed, there is already extended conjugation over five aromatic rings in CDTBT. However, there might be additional reasons for the small bandgap reduction in PCDTBT. For a strong reduction of bandgap upon polymerization, the orbitals involved in the first transition (mainly HOMO and LUMO) must disperse efficiently into the $\pi$-conjugated system. ${ }^{13}$ This requires strong coupling of the repeat units and a strong concentration of the electron density of the involved orbitals on the linking positions. The charge transfer character leading to partial electron localization on the BT acceptor units (partly outside the backbone) in the first excited state might therefore also be at the origin of the relatively low bandgap reduction. Indeed, it has been reported that the absorption maxima of dTBT oligomers shift to lower energy with increasing chain length to a lesser extent than the ones of oligomers in which the acceptor is within the backbone. ${ }^{13}$ Nevertheless, it must be pointed out here that the optical bandgap of the dTBT polymer (polydTBT) is only about $1.2 \mathrm{eV}$, according to solid-state experimental data ${ }^{59}$ and to TD-DFT calculations. ${ }^{80}$ This data is not directly comparable to our experimental work done in dilute solution (where intermolecular effects are negligible and only intrinsic electronic properties are probed). It is still very noteworthy that the PCDTBT polymer appears to have a surprisingly higher bandgap $(1.9 \mathrm{eV})$ than polydTBT $(1.2 \mathrm{eV})$, although the situation is exactly the opposite for the corresponding repeat units. We therefore tentatively suggest a third reason for the small bandgap reduction in dissolved PCDTBT: the loss of coplanarity introduced in the polymer backbone by the presence of the CB moiety (we calculated a $26^{\circ}$ twist angle with the dTBT plane). Twist angles between the $\mathrm{D}$ and $\mathrm{A}$ moieties along the polymer backbone have been shown to strongly affect the photophysics of D-A copolymers. ${ }^{52}$ Also, the reduction of polymer coplanarity provoked by side chains on the thiophene units of dTBT-containing D-A copolymers shifts the absorption spectra to higher energy. ${ }^{33,82}$

Finally, the experimental and theoretical results presented here, as well as the previously reported calculations, ${ }^{27}$ are in strong contradiction with the concept that the first absorption band in PCDTBT and chemically very similar polymers is a localized charge transfer transition, while the second band is a completely delocalized $\pi-\pi^{*}$ transition. ${ }^{36}$ We found that both 
bands have partial charge transfer character. We insist on the word partial, since all dominant transitions involve $\pi$-orbitals that extend over the polymer molecule, not orbitals that are localized on a single donor or acceptor moiety. The charge transfer implies concentration of electron density toward several of the electron-accepting units, which are themselves distributed over the whole chain. It is therefore not in contradiction with initial delocalization of the electronic states that are directly excited in the first and second absorption bands. There is increasing experimental evidence that the primary photoexcitation in conjugated polymers is delocalized along the polymer backbone and that it then self-localizes on the ultrafast time scale, ${ }^{16-22}$ including for PCDTBT. ${ }^{24}$ This self-localization in the excited state, driven by geometrical relaxation due to strong coupling to phonon modes, is partially responsible for the Stokes shift of the steady-state spectra. We observed a reduction of the Stokes shift when going from dTBT to CDTBT and to PCDTBT, caused by a lesser red shift of the emission compared to the absorption bands. We are currently conducting time-resolved experiments to establish whether this observation is linked to self-localization in the more conjugated molecules.

3.5. CDTBT and PCDTBT in the Solid State. The results presented until now were recorded in very dilute solutions or calculated in the gas phase. The orbital levels and optical transition energies of the molecules in the solid state can be strongly affected by additional intermolecular packing effects (aggregation). In Figure 2, the steady-state spectra of CDTBT and PCDTBT as solid thin films are shown in gray. For CDTBT, the shape of the solid-state absorption spectrum resembles the ones recorded in solution, except that the three absorption bands are slightly red-shifted (by about $1000 \mathrm{~cm}^{-1}$ compared to ACN). The absorption edge is now at 16100 $\mathrm{cm}^{-1}(2.0 \mathrm{eV}$, thus $0.1 \mathrm{eV}$ lower than in solution) and the first absorption maximum at $19120 \mathrm{~cm}^{-1}$. The second absorption band in the thin film has a shoulder on the low-energy side (as was observed for dTBT, but not for CDTBT in solution). There also seems to be an intensity enhancement of the two high-energy bands, but this could be due to light scattering from the film. The emission spectrum of CDTBT film has a symmetrical shape like the ones recorded in polar solvents. It is more red-shifted than in ACN (by $470 \mathrm{~cm}^{-1}$ ), with a maximum at $13880 \mathrm{~cm}^{-1}$ and a Stokes shift of $5240 \mathrm{~cm}^{-1}$. From those results (small shifts), it appears that the packing effects of CDTBT in the solid state are not very important (amorphous molecular arrangement), so that the energy levels of the transitions are hardly affected by intermolecular interaction.

The solid-state absorption spectrum of PCDTBT is even more similar to the one measured in DCB solution (Figure 2D). The first absorption band broadens and its maximum (at $17200 \mathrm{~cm}^{-1}$ ) is slightly red-shifted in the polymer thin film. As in CDTBT, the bandgap is $0.1 \mathrm{eV}$ lower in the solid state (at $\left.1.8 \mathrm{eV}, 14825 \mathrm{~cm}^{-1}\right)$. The second absorption band of the polymer is even more similar in thin film and solution. A third absorption band in PCDTBT is only accessible in the solid state. It is at the same spectral position as in the repeat unit, although it appears slightly broader with a lower relative intensity. The emission spectrum of PCDTBT peaks at 13810 $\mathrm{cm}^{-1}$ in both DCB and thin film, but the blue edge is at higher energy in solution. There is only a very slight shift $\left(<100 \mathrm{~cm}^{-1}\right)$ between the solid-state emission spectra of PCDTBT and CDTBT. The polymer spectrum is very slightly narrower and less symmetrical. Previous time-resolved fluorescence measure- ments have also revealed very similar dynamics in dissolved and solid PCDTBT during the first 10 ps following excitation, confirming a comparable chain conformation and intermolecular arrangement. ${ }^{24}$ Although aggregation of PCDTBT has been reported in more concentrated solution, and X-ray diffraction revealed some kind of ordering in the thin films, ${ }^{34}$ PCDTBT is generally considered an amorphous polymer in the solid state, in contrast to more crystalline materials such as P3HT. The amorphous packing is also more consistent with the similarity of the steady-state spectra in solution and thin film reported here. On the basis of the very small permanent dipole moment in the ground state, which is responsible for the weak absorption solvatochromomism, we can also conclude that intermolecular permanent dipole-dipole interactions do not play a major role in solid CDTBT and PCDTBT (induced dipole interactions should be more important).

Although we have shown very similar optical properties in CDTBT and PCDTBT, the importance of polymerization becomes clear when the electrical properties (conductivity, photoconductivity) of the solid-state compounds are considered (Figure 6). It is known that photoconductivity results from
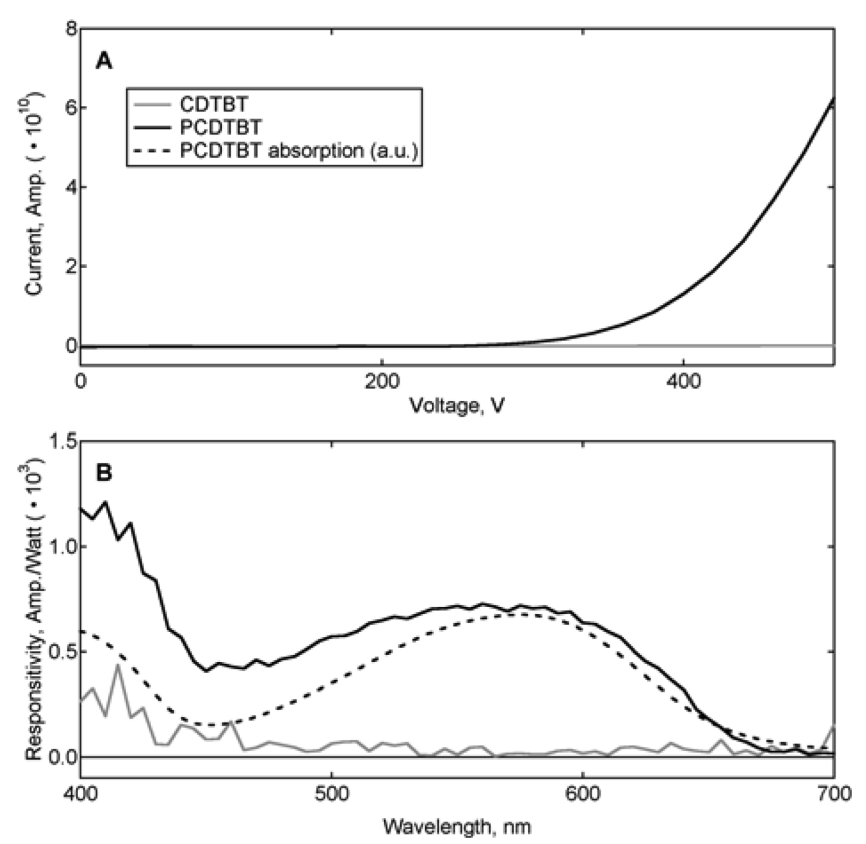

Figure 6. (A) Current-voltage curves recorded for CDTBT and PCDTBT thin films in the dark, in planar Auston switch configuration with gold electrodes separated by a $50 \mu \mathrm{m}$ gap. (B) Photoresponsitivity spectra of the same samples recorded with an applied bias of $400 \mathrm{~V}$ for PCDTBT and $500 \mathrm{~V}$ for CDTBT.

excitation of pristine conjugated polymer films. The mechanism of mobile charge carrier formation, which is at the origin of macroscopic photocurrent, is still matter of some debate (direct or via dissociation of (hot) excitons). ${ }^{25,83-87}$ It occurs on the ultrafast time scale $(<100-200 \mathrm{fs})$ and should therefore involve the nonrelaxed excited state of the polymer. It seems plausible that both the delocalization in the directly excited state and the intermolecular packing of the polymer chains play a major role in charge generation efficiency. We suggest here in more general terms that mobile charge carriers appear when the negative and positive part (electron and hole) of the delocalized primary photoexcitation self-localize as polarons (defined as a 
charge coupled to a structural distortion) on separate polymer chains.

Steady-state photoconductivity measurements give an overall appreciation of free charge carrier photogeneration, lifetime, and transport in a solid-state material. As expected, we observe photocurrent for a pristine PCDTBT film, shown in Figure 6B. The photoresponsitivity spectrum (photocurrent per watt of incident light with an applied bias of $400 \mathrm{~V}$ ) follows the shape of the absorption spectrum (dashed line). In excellent agreement with literature, ${ }^{88}$ relatively more photocurrent is obtained in the second, higher energy absorption band. This is true even if the artifact of apparent response near $400 \mathrm{~nm}$, caused by the low reference signal from the reference detector, is subtracted. We ascribe this effect to higher delocalization following excitation in the second band, which favors charge splitting of the photoexcitation into charges between neighboring polymer chains. We could clearly show this higher delocalization in the second band by analysis of the optical transitions in the repeat unit (section 3.3). It is also consistent with the previously published TD-DFT calculations on the PCDTBT tetramer (section 3.4) ${ }^{27}$ In contrast to PCDTBT, its repeat unit CDTBT shows no photocurrent response even at an applied bias voltage of $500 \mathrm{~V}$ (Figure 6B). Note that the apparent response near $400 \mathrm{~nm}$ is an artifact. This could indicate poor generation of long-lived charge carriers in CDTBT, in spite of the partial charge transfer character of the transitions and electron delocalization in a relatively large molecule. The extended conjugation found only in the polymer seems to be necessary for formation of free polarons on separate molecules, which confirms the importance of the implied higher initial delocalization.

Of course, the packing in the solid state must also be appropriate for the carrier separation and transport to the electrodes. As discussed above, CDTBT and PCDTBT are rather amorphous in the solid state. Nevertheless, the molecular packing in solid-state PCDTBT is sufficient for a small yield of photocurrent and for a reported hole mobility of $10^{-3} \mathrm{~cm}^{2} \mathrm{~V}^{-1}$ $\mathrm{s}^{-1} \cdot{ }^{34}$ Given the very similar chemical nature, we would have expected similar intermolecular interactions in CDTBT. However, the current-voltage curve recorded in the dark (Figure 6A) shows that PCDTBT conducts injected charges when the applied voltage exceeds $300 \mathrm{~V}$, while practically no dark current is observed for CDTBT. This indicates that the electrical properties and intermolecular packing of CDTBT in the solid state do not allow efficient conduction of charges, pointing to significant differences compared to the polymer and also explaining the absence of photocurrent. This result is also the main reason for the strong reduction of short circuit current that we observed when incorporating even very small amounts of CDTBT into PCDTBT:fullerene solar cells in order to extend the active layer absorption spectrum (data not shown).

\section{SUMMARY AND CONCLUSIONS}

Recently, conjugated polymers with alternating electronwithdrawing and electron-donating groups along their backbone ( $\mathrm{D}-\mathrm{A}$ copolymers) have yielded very high power conversion efficiency in bulk heterojunction solar cells. It is believed that charge transfer within the repeat unit lowers the bandgap for better harvesting of the solar spectrum. However, the implications of this internal charge transfer character are not clear. In an effort to understand how the bandgap is reduced in a typical D-A copolymer, we decided to break down the problem by breaking down the polymer. We report here the synthesis of CDTBT, which is the isolated repeat unit of the very successful PCDTBT polymer. We have investigated the spectroscopic and electrochemical properties of PCDTBT, CDTBT, and of the separate donor (CB) and acceptor (dTBT) moieties in solution and in the solid state. The results are interpreted using computational methods (time-dependent DFT simulations). This has allowed a detailed elucidation of the electronic structure of PCDTBT.

A significant result is that the two broad bands that characterize the absorption spectrum of PCDTBT in the visible range are each dominated by only one transition, involving partial charge transfer character and some delocalization in the directly excited state (more pronounced in the second band). Since dTBT is already a D-A-D triad of thiophenes and BT, the structure of PCDTBT is more complex than an alternating $\mathrm{D}-\mathrm{A}$ chain. We found that there is strong mixing (electronic coupling) of the molecular orbitals of the separate $\mathrm{CB}$, thiophene, and $\mathrm{BT}$ segments in the CDTBT repeat unit as well as in the polymer. The main reduction of bandgap compared to those segments (which all absorb in the ultraviolet range) occurs already in dTBT. The dTBT moiety is also at the origin of the partial charge transfer character in the dominant transitions of PCDTBT, and it is responsible for the typical "camel back" shape of the polymer absorption spectrum. In $\mathrm{dTBT}$, both dominant transitions concentrate some electron density toward the central BT unit, but some delocalization over the molecule is maintained for both the hole and the electron wave functions.

Covalently attached $\mathrm{CB}$ extends the conjugation along the molecular backbone of CDTBT (which reduces the transition energy and increases the oscillator strength of the transitions), rather than acting as a localized donor unit. We found a small permanent dipole moment in the ground state of CDTBT, meaning that dipole-dipole interactions due to polarized repeat units do not play a major role in solid-state packing of the polymer. Also, the partial charge transfer during excitation is not sufficient to cause any significant dependence of the first two absorption bands in CDTBT on solvent polarity. When CDTBT is polymerized, a weak reduction of bandgap $(0.2 \mathrm{eV})$ and increase in oscillator strength result from the extended conjugation of the molecular orbitals involved in the dominant transitions along the polymer backbone (the same trend as when $\mathrm{CB}$ is attached to $\mathrm{dTBT}$ ). The reasons for the relatively small spectral red-shift in the polymer might include the already extended conjugation in the large repeat unit (it has 5 aromatic rings), the concentration of electron density on the thiadiazole units outside the molecular backbone during the dominant transitions, or loss of coplanarity in the polymer chain introduced by the CB moiety.

We want to attract particular attention to the fact that the charge transfer during excitation of PCDTBT in the first two absorption bands is only partial, since all dominant transitions involve $\pi$-orbitals that extend over the polymer chain, not orbitals that are localized on a single donor or acceptor moiety. The charge transfer concept in the D-A copolymer is therefore not in contradiction with initial delocalization of the electronic states that are directly excited by light absorption. ${ }^{16-22}$ It is this delocalization along the backbone that gives conjugated polymers their special properties, ${ }^{1,2}$ and we can conclude that they are not lost in PCDTBT. We suggest here that the initial delocalization favors splitting of the photoexcitation into charges between neighboring polymer chains in the solid state, which is the origin of macroscopic photoconductivity in 
pristine conjugated polymers. We demonstrated higher delocalization in the transition responsible for the second absorption band of PCDTBT, which explains the higher photocurrent yield when this band is excited compared to the first one. Insufficient delocalization in the excited states of unpolymerized CDTBT, together with low electrical conductivity, account for the absence of photocurrent in the solidstate repeat unit.

The initially delocalized photoexcitation in conjugated polymers rapidly self-localizes to a smaller number of repeat units. ${ }^{16-22}$ We observed here a strong charge transfer character in the emitting state of dTBT and CDTBT (implying localization of the charges during relaxation), leading to a strong dependence of the fluorescence spectrum on solvent polarity. We are currently investigating the excited-state relaxation in the two compounds using ultrafast time-resolved spectroscopy in order to elucidate whether the relaxation processes that increase the charge transfer character following excitation can be related to the self-localization observed in polymers. We have recently pointed out that the short-lived delocalized photoexcitation in conjugated polymers (PCDTBT and $\mathrm{P} 3 \mathrm{HT}$ ) might be responsible for the ultrafast charge separation observed in bulk heterojunction polymer:fullerene solar cells. ${ }^{16,24}$ Based on the relaxation time scales measured in the polymers, we concluded that the charge separation in polymer:fullerene blends must occur before the photoexcitation self-localizes. The results presented here are therefore not only highly important for a better understanding of $\mathrm{D}-\mathrm{A}$ copolymers in general but can also potentially guide the strategic development of future photovoltaic materials in view of the importance of initial delocalization for the functioning of bulk heterojunction solar cells.

\section{ASSOCIATED CONTENT}

\section{S Supporting Information}

Additional figures and tables as mentioned in the text; details of refs $6,25,33,42,70$, and 72 . This material is available free of charge via the Internet at http://pubs.acs.org.

\section{AUTHOR INFORMATION}

\section{Corresponding Author}

*E-mail: natalie.banerji@epfl.ch.

\section{Present Addresses}

${ }^{\perp}$ Institute of Chemical Sciences \& Engineering, Ecole Polytechnique Fédérale de Lausanne (EPFL), SB ISIC GRMO, Station 6, CH-1015 Lausanne, Switzerland.

\#Department of Materials Physics, Dong-A University, Busan 604-714, South Korea.

\section{Notes}

The authors declare no competing financial interest.

\section{ACKNOWLEDGMENTS}

N.B. thanks the Swiss National Science Foundation for Fellowship support (fellowship for prospective researchers PBGEP2-125859). S.V. acknowledges support by the Karlsruhe House of Young Scientists (KHYS) and the DFG Center for Functional Nanostructures (CFN). Dr. Sarah Cowan, Dr. Jonathan Yuen, Dr. Daniel Moses, Dr. Serge Beaupré, Prof. Eric Vauthey, Prof. Tomasz Wesolowski, and Prof. Fred Wudl are acknowledged for fruitful discussions and for use of their equipment. Research at UCSB was carried out with support from the Department of Energy under a grant titled "Charge
Recombination, Transport Dynamics, and Interfacial Effects in Organic Solar Cells"; DOE \#DE-FG02-08ER46535.

\section{REFERENCES}

(1) Heeger, A. J.; Sariciftci, N. S.; Namdas, E. B. Semiconducting and Metallic Polymers; Oxford University Press: Oxford, UK, 2010.

(2) Heeger, A. J. Chem. Soc. Rev. 2010, 39, 2354-2371.

(3) Yuen, J. D.; Kumar, R.; Zakhidov, D.; Seifter, J.; Lim, B.; Heeger,

A. J.; Wudl, F. Adv. Mater. (Weinheim, Ger.) 2011, 23, 3780-3785.

(4) Sonar, P.; Singh, S. P.; Li, Y.; Soh, M. S.; Dodabalapur, A. Adv. Mater. (Weinheim, Ger.) 2010, 22, 5409-5413.

(5) Bijleveld, J. C.; Zoombelt, A. P.; Mathijssen, S. G. J.; Wienk, M. M.; Turbiez, M.; de Leeuw, D. M.; Janssen, R. A. J. J. Am. Chem. Soc. 2009, 131, 16616-16617.

(6) Lu, J. P.; Chu, T. Y.; Beaupre, S.; Zhang, Y. G.; Pouliot, J. R.; Wakim, S.; Zhou, J. Y.; Leclerc, M.; Li, Z.; Ding, J. F.; et al. J. Am. Chem. Soc. 2011, 133, 4250-4253.

(7) Heeger, A. J.; Sun, Y. M.; Takacs, C. J.; Cowan, S. R.; Seo, J. H.; Gong, X.; Roy, A. Adv. Mater. (Weinheim, Ger.) 2011, 23, 2226-2230.

(8) Yu, L. P.; Liang, Y. Y.; Xu, Z.; Xia, J. B.; Tsai, S. T.; Wu, Y.; Li, G.; Ray, C. Adv. Mater. (Weinheim, Ger.) 2010, 22, E135-E138.

(9) Chen, H. Y.; Hou, J. H.; Zhang, S. Q.; Liang, Y. Y.; Yang, G. W.; Yang, Y.; Yu, L. P.; Wu, Y.; Li, G. Nat. Photonics 2009, 3, 649-653.

(10) Bundgaard, E.; Krebs, F. C. Sol. Energy Mater. Sol. Cells 2007, 91, 954-985.

(11) Scharber, M. C.; Muehlbacher, D.; Koppe, M.; Denk, P.; Waldauf, C.; Heeger, A. J.; Brabec, C. J. Adv. Mater. (Weinheim, Ger.) 2006, 18, 789-794.

(12) Ajayaghosh, A. Chem. Soc. Rev. 2003, 32, 181-191.

(13) van Mullekom, H. A. M.; Vekemans, J. A. J. M.; Havinga, E. E.; Meijer, E. W. Mater. Sci. Eng., R 2001, 32, 1-40.

(14) Scheblykin, I. G.; Yartsev, A.; Pullerits, T.; Gulbinas, V.; Sundstrom, V. J. Phys. Chem. B 2007, 111, 6303-6321.

(15) Sariciftci, N. S. Primary Photoexcitations in Conjugated Polymers: Molecular Exciton versus Semiconductor Band Model; World Scientific: Singapore, 1997.

(16) Banerii, N.; Cowan, S.; Vauthey, E.; Heeger, A. J. J. Phys. Chem. C 2011, 115, 9726-9739.

(17) Collini, E.; Scholes, G. D. Science (Washington, DC, U. S.) 2009, 323, 369-373.

(18) Wells, N. P.; Blank, D. A. Phys. Rev. Lett. 2008, 100, 086403.

(19) Ruseckas, A.; Wood, P.; Samuel, I. D. W.; Webster, G. R.; Mitchell, W. J.; Burn, P. L.; Sundstrom, V. Phys. Rev. B 2005, 72, 115214.

(20) Yang, X. J.; Dykstra, T. E.; Scholes, G. D. Phys. Rev. B 2005, 71, 045203.

(21) Dykstra, T. E.; Kovalevskij, V.; Yang, X. J.; Scholes, G. D. Chem. Phys. 2005, 318, 21-32.

(22) Milota, F.; Sperling, J.; Tortschanoff, A.; Szocs, V.; Kuna, L.; Kauffmann, H. F. J. Lumin. 2004, 108, 205-209.

(23) Beaujuge, P. M.; Amb, C. M.; Reynolds, J. R. Acc. Chem. Res. 2010, 43, 1396-1407.

(24) Banerji, N.; Cowan, S.; Leclerc, M.; Vauthey, E.; Heeger, A. J. J. Am. Chem. Soc. 2010, 132, 17459-17470.

(25) Westerling, M.; Aarnio, H.; Osterbacka, R.; Stubb, H.; King, S. M.; Monkman, A. P.; Andersson, M. R.; Jespersen, K.; Kesti, T.; Yartsev, A.; et al. Phys. Rev. B 2007, 75, 224306.

(26) Lin, Z.; Bjorgaard, J.; Gul, Y. A.; Iyer, A.; Koese, M. E. RSC Adv. 2012, 2, 642-651.

(27) Risko, C.; McGehee, M. D.; Bredas, J. L. Chem. Sci. 2011, 2, 1200-1218.

(28) Janesko, B. G. J. Chem. Phys. 2011, 134, 184105.

(29) Canestraro, C. D.; Rodrigues, P. C.; Marchiori, C. F. N.; Schneider, C. B.; Akcelrud, L.; Koehler, M.; Roman, L. S. Sol. Energy Mater. Sol. Cells 2011, 95, 2287-2294.

(30) Wang, Y.; Peng, Q.; Hou, Q.; Zhao, K.; Liang, Y.; Li, B. Theor. Chem. Acc. 2011, 129, 257-270.

(31) Ou, P.; Shen, W.; He, R.; Xie, X.; Zeng, C.; Li, M. Polym. Int. 2011, 60, 1408-1418. 
(32) Beaupré, S.; Belletête, M.; Durocher, G.; Leclerc, M. Macromol. Theory Simul. 2011, 20, 13-18.

(33) Friend, R. H.; Oberhumer, P. M.; Huang, Y. S.; Massip, S.; James, D. T.; Tu, G. L.; Albert-Seifried, S.; Beljonne, D.; Cornil, J.; Kim, J. S.; et al. J. Chem. Phys. 2011, 134, 114901.

(34) Blouin, N.; Michaud, A.; Gendron, D.; Wakim, S.; Blair, E.; Neagu-Plesu, R.; Belletete, M.; Durocher, G.; Tao, Y.; Leclerc, M. J. Am. Chem. Soc. 2008, 130, 732-742.

(35) Gierschner, J.; Cornil, J.; Egelhaaf, H. J. Adv. Mater. (Weinheim, Ger.) 2007, 19, 173-191.

(36) Jespersen, K. G.; Beenken, W. J. D.; Zaushitsyn, Y.; Yartsev, A.; Andersson, M.; Pullerits, T.; Sundstrom, V. J. Chem. Phys. 2004, 121, 12613-12617.

(37) Banerji, N.; Bhosale, S. V.; Petkova, I.; Langford, S. J.; Vauthey, E. Phys. Chem. Chem. Phys. 2011, 13, 1019-1029.

(38) Janssen, R. A. J.; Karsten, B. P.; Viani, L.; Gierschner, J.; Cornil, J. J. Phys. Chem. A 2009, 113, 10343-10350.

(39) Banerji, N.; Angulo, G.; Barabanov, I.; Vauthey, E. J. Phys. Chem. A 2008, 112, 9665-9674.

(40) Wasielewski, M. R.; Niemczyk, M. P.; Svec, W. A.; Pewitt, E. B. J. Am. Chem. Soc. 1985, 107, 1080-1082.

(41) Banerji, N.; Duvanel, G.; Perez-Velasco, A.; Maity, S.; Sakai, N.; Matile, S.; Vauthey, E. J. Phys. Chem. A 2009, 113, 8202-8212.

(42) Bhosale, S.; Sisson, A. L.; Talukdar, P.; Fuerstenberg, A.; Banerji, N.; Vauthey, E.; Bollot, G.; Mareda, J.; Roeger, C.; Wuerthner, F.; et al. Science (Washington, DC, U. S.) 2006, 313, 84-86.

(43) Rybtchinski, B.; Sinks, L. E.; Wasielewski, M. R. J. Am. Chem. Soc. 2004, 126, 12268-12269.

(44) Guldi, D. M. Chem. Soc. Rev. 2002, 31, 22-36.

(45) Imahori, H.; Guldi, D. M.; Tamaki, K.; Yoshida, Y.; Luo, C. P.; Sakata, Y.; Fukuzumi, S. J. Am. Chem. Soc. 2001, 123, 6617-6628.

(46) SteinbergYfrach, G.; Liddell, P. A.; Hung, S. C.; Moore, A. L.; Gust, D.; Moore, T. A. Nature 1997, 385, 239-241.

(47) Wu, Z. L.; Fan, B. H.; Xue, F.; Adachi, C.; Ouyang, J. Y. Sol. Energy Mater. Sol. Cells 2010, 94, 2230-2237.

(48) Tang, W. H.; Hai, J. F.; Dai, Y.; Huang, Z. J.; Lu, B. Q.; Yuan, F.; Tang, J. A.; Zhang, F. J. Sol. Energy Mater. Sol. Cells 2010, 94, 19631979.

(49) Lee, Y. S.; Li, J. C.; Lee, H. Y.; Lee, S. H.; Zong, K.; Jin, S. H. Synth. Met. 2009, 159, 201-208.

(50) Walker, B.; Tamayo, A. B.; Dang, X.-D.; Zalar, P.; Seo, J. H.; Garcia, A.; Tantiwiwat, M.; Nguyen, T.-Q. Adv. Funct. Mater. 2009, 19, 3063-3069.

(51) Chen, C.-H.; Chen, W.-H.; Liu, Y.-H.; Lim, T.-S.; Luh, T.-Y. Chem.-Eur. J. 2012, 18, 347-354.

(52) Habuchi, S.; Fujita, H.; Michinobu, T.; Vacha, M. J. Phys. Chem. B 2011, 115, 14404-14415.

(53) Porzio, W.; Destri, S.; Pasini, M.; Giovanella, U.; Ragazzi, M.; Scavia, G.; Kotowski, D.; Zotti, G.; Vercelli, B. New J. Chem. 2010, 34, 1961-1973.

(54) Chen, L. X.; Liang, V. Y.; Feng, D. Q.; Guo, J. C.; Szarko, J. M.;

Ray, C.; Yu, L. P. Macromolecules 2009, 42, 1091-1098.

(55) Karsten, B. P.; Viani, L.; Gierschner, J.; Cornil, J.; Janssen, R. A. J. J. Phys. Chem. A 2008, 112, 10764-10773.

(56) Jenekhe, S. A.; Lu, L. D.; Alam, M. M. Macromolecules 2001, 34, $7315-7324$

(57) Cui, Y. T.; Zhang, X. J.; Jenekhe, S. A. Macromolecules 1999, 32, 3824-3826.

(58) Zhang, Q. T.; Tour, J. M. J. Am. Chem. Soc. 1997, 119, 50655066.

(59) Kitamura, C.; Tanaka, S.; Yamashita, Y. Chem. Mater. 1996, 8, 570-578.

(60) Blouin, N.; Michaud, A.; Leclerc, M. Adv. Mater. (Weinheim, Ger.) 2007, 19, 2295-2300.

(61) Blouin, N.; Leclerc, M. Acc. Chem. Res. 2008, 41, 1110-1119.

(62) Scharber, M. C.; Wuhlbacher, D.; Koppe, M.; Denk, P.; Waldauf, C.; Heeger, A. J.; Brabec, C. L. Adv. Mater. (Weinheim, Ger.) 2006, 18, 789-794.
(63) Park, S. H.; Roy, A.; Beaupre, S.; Cho, S.; Coates, N.; Moon, J. S.; Moses, D.; Leclerc, M.; Lee, K.; Heeger, A. J. Nat. Photonics 2009, 3, 297-302.

(64) Sun, Y.; Takacs, C. J.; Cowan, S. R.; Seo, J. H.; Gong, X.; Roy, A.; Heeger, A. J. Adv. Mater. (Weinheim, Ger.) 2011, 23, 2226-2230.

(65) Boudreault, P. L. T. Synthèse et caractérisation de nouveaux semi-conducteurs organiques èa base de carbazole et d'indolo(3,2-b) carbazole. PhD Thesis, Université Laval, 2010.

(66) deMello, J. C.; Wittmann, H. F.; Friend, R. H. Adv. Mater. (Weinheim, Ger.) 1997, 9, 230-232.

(67) Angulo, G.; Grampp, G.; Rosspeintner, A. Spectrochim. Acta, Part A 2006, 65A, 727-731.

(68) Forrest, S. R.; D’Andrade, B. W.; Datta, S.; Djurovich, P.; Polikarpov, E.; Thompson, M. E. Org. Electron. 2005, 6, 11-20.

(69) Becke, A. D. J. Chem. Phys. 1993, 98, 5648-5652.

(70) Frisch, M. J.; Trucks, G. W.; Schlegel, H. B.; Scuseria, G. E.; Robb, M. A.; Cheeseman, J. R.; Montgomery, J. A.; Vreven, T.; Kudin, K. N.; Burant, J. C.; et al. Gaussian 03, Revision B.03; Gaussian, Inc.: Wallingford, CT, 2004.

(71) Varetto, U. Swiss National Supercomputing Centre, Manno, Switzerland.

(72) Frisch, M. J.; Trucks, G. W.; Schlegel, H. B.; Scuseria, G. E.; Robb, M. A.; Cheeseman, J. R.; Scalmani, G.; Barone, V.; Mennucci, B.; Petersson, G. A.; et al. Gaussian 09, Revision C.1; Gaussian, Inc.: Wallingford, CT, 2009.

(73) Brunner, K.; van Dijken, A.; Borner, H.; Bastiaansen, J. J. A. M.; Kiggen, N. M. M.; Langeveld, B. M. W. J. Am. Chem. Soc. 2004, 126, 6035-6042.

(74) Gordon, R. D.; Yang, R. F. J. Mol. Spectrosc. 1971, 39, 295-320.

(75) Henry, B. R.; Morrison, J. D. J. Mol. Spectrosc. 1975, 55, 311318.

(76) Wan, J.; Hada, M.; Ehara, M.; Nakatsuji, H. J. Chem. Phys. 2001, $114,842-850$.

(77) Hush, N. S. In Progress in Inorganic Chemistry; John Wiley \& Sons, Inc.: New York, 1967; pp 391-444.

(78) Suppan, P. Chemistry and Light; The Royal Society of Chemistry: Cambridge, 1994.

(79) Suppan, P. J. Photochem. Photobiol., A 1990, 50, 293-330.

(80) Ozen, A. S.; Atilgan, C.; Sonmez, G. J. Phys. Chem. C 2007, 111, $16362-16371$.

(81) Edward, J. T. J. Chem. Educ. 1970, 47, 261-270.

(82) Kim, J.; Kwon, Y. S.; Shin, W. S.; Moon, S. J.; Park, T. Macromolecules 2011, 44, 1909-1919.

(83) Kohler, A.; dos Santos, D. A.; Beljonne, D.; Shuai, Z.; Bredas, J. L.; Holmes, A. B.; Kraus, A.; Mullen, K.; Friend, R. H. Nature 1998, 392, 903-906.

(84) Silva, C.; Dhoot, A. S.; Russell, D. M.; Stevens, M. A.; Arias, A. C.; MacKenzie, J. D.; Greenham, N. C.; Friend, R. H.; Setayesh, S.; Mullen, K. Phys. Rev. B 2001, 64, 125211.

(85) Moses, D.; Soci, C.; Miranda, P.; Heeger, A. J. Chem. Phys. Lett. 2001, 350, 531-536.

(86) Miranda, P. B.; Moses, D.; Heeger, A. J. Phys. Rev. B 2001, 6408, 081201.

(87) Gulbinas, V.; Zaushitsyn, Y.; Bassler, H.; Yartsev, A.; Sundstrom, V. Phys. Rev. B 2004, 70, 035215.

(88) Tong, M. H.; Coates, N. E.; Moses, D.; Heeger, A. J.; Beaupre, S.; Leclerc, M. Phys. Rev. B 2010, 81, 125210. 